\title{
Isogeometric Divergence-Conforming Variational Multiscale Formulation of Incompressible Turbulent Flows
}

\author{
T.M. van Opstal ${ }^{1}$, J. Yan², C. Coley $^{3}$, J.A. Evans ${ }^{3}$, T. Kvamsdal ${ }^{1}$, and Y. Bazilevs*2 \\ ${ }^{1}$ Department of Mathematical Sciences, Norwegian University of Science and Technology \\ ${ }^{2}$ Department of Structural Engineering, University of California, San Diego \\ ${ }^{3}$ Department of Aerospace Engineering, University of Colorado, Boulder
}

September 30, 2016

\begin{abstract}
A new residual-based variational multiscale (RBVMS) formulation for incompressible turbulent flows is proposed that is suitable for discretization using divergence-conforming B-splines. The proposed methodology results in a pointwise satisfaction of the zero-divergence constraint on the discrete velocity field. The velocity fine scales are residual-driven and constructed in a manner that is consistent with the divergence-free constraint on the discrete velocity solution. The resulting formulation is tested on laminar- and turbulent-flow benchmark problems showing excellent stability and accuracy characteristics in both regimes.
\end{abstract}

Keywords: IGA; VMS; Divergence-conforming B-splines; Turbulent flows; Incompressible flows

\section{Introduction}

The basic theory of variational multiscale (VMS) methods was put forth in [34]. In the same reference, well-known stabilized methods, such as the SUPG and PSPG techniques [15, 48], were first identified as multiscale methods derivable on the basis of the VMS theory. The possibility of using VMS for subgrid-scale modeling in large-eddy simulation (LES) of incompressible turbulent flows was first discussed in [36]. More recently, in [5], based on the VMS framework, the authors developed a residual-based variational multiscale (RBVMS) formulation for LES of incompressible turbulent flows. This and later references [9, 10,30] showed that RBVMS solutions converged rapidly to the direct numerical simulation (DNS) results and yielded LES-like solutions on intermediate meshes. These features are found to be particularly attractive for predictive simulation

${ }^{*}$ Corresponding author. E-mail: yuri@ucsd.edu

(C) 2016. This manuscript version is made available under the Elsevier user license http://www.elsevier.com/open-access/userlicense/1.0/ 
of turbulent flows in engineering applications, because in many cases the flow regime at a given location in space is often not known a priori, yet the flow solution needs to be computed with sufficient accuracy. It should also be noted that the RBVMS concept was explored in an earlier reference [18] in the context of finite volume methods.

In an effort to improve the accuracy of RBVMS in the presence of unresolved (or marginally resolved) boundary layers, weakly enforced no-slip conditions were introduced in [6]. The RBVMS formulation, in combination with weakly-enforced no-slip conditions, produced significant solution accuracy improvement as evidenced by the results obtained from a number of challenging turbulentflow test cases $[9,4]$ and engineering-scale simulations $[1,32,31]$.

Isogeometric Analysis (IGA), proposed in [35] as a computational methodology to better integrate Computer-Aided Design (CAD) and Finite Element Analysis (FEA) [20], rapidly became a driving force behind much of the recent developments in VMS methods for turbulent flows. In [5] the RBVMS formulation was discretized using IGA based on B-splines, yielding excellent perdegree-of-freedom accuracy of the discrete solutions. This additional accuracy was verified in [2] to be a consequence of the higher-order continuity of B-splines.

In [16], the structure and higher-order continuity of the B-spline basis were exploited to construct approximation spaces that form the so-called discrete de Rham complex [17]. An important consequence of this construction is that suitably chosen approximation spaces for incompressibleflow equations lead to a pointwise satisfaction of the zero-divergence constraint for the discrete velocity field, which is in contrast to other approaches that satisfy this condition only in a weak sense. This so-called divergence-conforming B-spline discretization was successfully employed to solve the Navier-Stokes equations of incompressible flows in the laminar regime using the Galerkin technique in $[29,28]$. Excellent solution accuracy and stability, especially using meshes with moderate resolution relative to the flow Reynolds number employed, were reported in the aforementioned references. These were attributed to the exact satisfaction of energy, helicity, and enstrophy balances in the semi-discrete setting, all stemming from a pointwise satisfaction of the zero-divergence constraint. Finally, it was argued that turbulent-flow simulations may benefit from accuracy and stability improvement of the divergence-conforming B-spline discretizations, which by and large motivates the developments in the present article.

This paper lays the groundwork for a new generation of methods for computational fluid dynamics (CFD) and turbulence modeling that combine the finest attributes of VMS and divergenceconforming B-spline discretizations. A new VMS formulation is derived that is residual-based and produces pointwise divergence-free discrete velocity solution, as advocated by the divergenceconforming B-spline paradigm. The use of higher-order continuous B-splines in the construction of the divergence-conforming basis, in addition to resulting in a pointwise satisfaction of the zerodivergence constraint, also gives rise to $H^{1}$-conforming discretizations, as required for the present setting.

In Section 2 we state the weak form of the Navier-Stokes equations of incompressible flows. In Section 3 we recall the construction of the divergence-conforming B-spline basis for the velocity and pressure unknowns. In Section 4 we derive a general form of the divergence-conforming VMS 
formulation. In Section 5 we develop the fine-scale velocity approximations. In Section 6 we briefly recall weak enforcement of no-slip boundary conditions and kinematic and traction compatibility for multi-patch discretizations. In Section 7 we present numerical examples. In Section 8 we draw conclusions.

\section{Navier-Stokes equations of incompressible flows}

Let $\Omega \subset \mathbb{R}^{d}, d=2,3$ denote the problem spatial domain with boundary $\partial \Omega$, and let $I:=(0, T), T \in$ $\mathbb{R}_{>0}$ the time interval of interest. The Navier-Stokes equations of viscous, unsteady, incompressible flows subject to homogeneous velocity boundary conditions may be stated as: Given $\nu \in \mathbb{R}_{>0}$, $f: \Omega \times I \rightarrow \mathbb{R}^{d}$, and $u_{0}: \Omega \rightarrow \mathbb{R}^{d}$, find $u: \Omega \times I \rightarrow \mathbb{R}^{d}$ and $p: \Omega \times I \rightarrow \mathbb{R}$, such that,

$$
\begin{cases}\partial_{t} u+u \cdot \nabla u+\nabla p-\nabla \cdot \nu \nabla^{s} u=f & \text { in } \Omega \times I, \\ \nabla \cdot u=0 & \text { in } \Omega \times I, \\ u=0 & \text { on } \partial \Omega \times I, \\ u=u_{0} & \text { on } \Omega \times\{0\} .\end{cases}
$$

Here $u$ and $p$ are the flow velocity and pressure, respectively, $u_{0}$ is the velocity initial data, $\nu$ is the kinematic viscosity, $f$ is the body force per unit mass, and $\nabla^{s}$ is the symmetric gradient. Note that because of the boundary conditions chosen, pressure is only determined up to an additive constant.

We introduce the spaces $V:=H_{0}^{1}(\Omega)$ and $Q:=L_{0}^{2}(\Omega)$, and state the weak formulation of the Navier-Stokes equations: Find $u \in V$ and $p \in Q$, such that

$$
B((u, p) ;(v, q))=F(v, q) \quad \forall(v, q) \in V \times Q,
$$

where

$$
\begin{aligned}
B((u, p) ;(v, q)) & =\left(\partial_{t} u, v\right)-(u \otimes u, \nabla v)+2 \nu\left(\nabla^{s} u, \nabla^{s} v\right) \\
& -(p, \nabla \cdot v)+(\nabla \cdot u, q) \\
F(v, q) & =(f, v) .
\end{aligned}
$$

In the above equations, $(\cdot, \cdot)$ denotes the usual $L^{2}$-inner product over $\Omega$. Note that, in the semilinear form given by Eq. (3), convection has been brought into the conservative form. Also note that in the definition of the pressure-solution and continuity-test-function spaces the subscript ' 0 ' denotes functions with zero mean over $\Omega$.

\section{Divergence-conforming B-splines}

Divergence-conforming B-splines were first introduced in [16], and recently extended to locallyrefined B-splines in [39]. Divergence-conforming B-splines were successfully applied to simulate 
Stokes, Darcy, and low-Reynolds-number incompressible flows using the Galerkin technique in [27, $28,29]$. In this section we give a concise overview of the divergence-conforming B-spline space construction.

\subsection{B-spline basics}

Univariate B-spline basis functions are constructed using a knot vector in $1 \mathrm{D}$, a non-decreasing set of coordinates in the parameter space written as $\Xi=\left\{\xi_{1}, \xi_{2}, \ldots, \xi_{n+p+1}\right\}$, where $\xi_{i} \in \mathcal{R}$ is the $i^{t h}$ knot, $i$ is the knot index, $i=1,2, \ldots, n+p+1, \quad p$ is the polynomial order, and $n$ is the number of basis functions. Knots partition the parameter space into elements. For a given knot vector, B-spline basis functions are defined recursively starting with piecewise constants $(p=0)$ :

$$
N_{i, 0}(\xi)= \begin{cases}1 & \text { if } \xi_{i} \leq \xi<\xi_{i+1} \\ 0 & \text { otherwise }\end{cases}
$$

For $p=1,2,3, \ldots$, they are defined by

$$
N_{i, p}(\xi)=\frac{\xi-\xi_{i}}{\xi_{i+p}-\xi_{i}} N_{i, p-1}(\xi)+\frac{\xi_{i+p+1}-\xi}{\xi_{i+p+1}-\xi_{i+1}} N_{i+1, p-1}(\xi)
$$

which is the Cox-de Boor recursion formula (see [21, 23]). The support of B-spline functions of order $p$ is always $p+1$ knot spans.

In $2 \mathrm{D}$ and $3 \mathrm{D}$, B-spline basis functions are given as follows:

$$
\begin{aligned}
B_{i, j}^{p, q}(\xi, \eta) & =N_{i, p}(\xi) M_{j, q}(\eta), \\
B_{i, j, k}^{p, q, r}(\xi, \eta, \zeta) & =N_{i, p}(\xi) M_{j, q}(\eta) L_{k, r}(\zeta) .
\end{aligned}
$$

Here $\Xi=\left\{\xi_{1}, \xi_{2}, \ldots, \xi_{n+p+1}\right\}, \mathcal{H}=\left\{\eta_{1}, \eta_{2}, \ldots, \eta_{m+q+1}\right\}$, and $\mathcal{Z}=\left\{\zeta_{1}, \zeta_{2}, \ldots, \zeta_{l+r+1}\right\}$ are the knot vectors. The B-spline basis is pointwise nonnegative, and forms a partition of unity. The number of continuous derivatives in a given parametric direction may be determined from the associated $1 \mathrm{D}$ knot vector and polynomial order.

Given a mesh of control points, $\left\{\mathrm{C}_{i, j}\right\}, i=1,2, \ldots, n, j=1,2, \ldots, m$, polynomial orders $p$ and $q$, and knot vectors $\Xi=\left\{\xi_{1}, \xi_{2}, \ldots, \xi_{n+p+1}\right\}$ and $\mathcal{H}=\left\{\eta_{1}, \eta_{2}, \ldots, \eta_{m+q+1}\right\}$, a B-spline surface is defined as

$$
\mathrm{S}(\xi, \eta)=\sum_{i=1}^{n} \sum_{j=1}^{m} B_{i, j}^{p, q}(\xi, \eta) \mathrm{C}_{i, j} .
$$

A B-spline volume is constructed in an analogous fashion. Given a control mesh $\left\{\mathrm{C}_{i, j, k}\right\}, i=$ $1,2, \ldots, n, j=1,2, \ldots, m, k=1,2, \ldots, l$, polynomial orders $p, q$, and $r$, and knot vectors $\Xi=$ 
$\left\{\xi_{1}, \xi_{2}, \ldots, \xi_{n+p+1}\right\}, \mathcal{H}=\left\{\eta_{1}, \eta_{2}, \ldots, \eta_{m+q+1}\right\}$, and $\mathcal{Z}=\left\{\zeta_{1}, \zeta_{2}, \ldots, \zeta_{l+r+1}\right\}$, a B-spline volume is defined as

$$
\mathrm{S}(\xi, \eta, \zeta)=\sum_{i=1}^{n} \sum_{j=1}^{m} \sum_{k=1}^{l} B_{i, j, k}^{p, q, r}(\xi, \eta, \zeta) \mathrm{C}_{i, j, k} .
$$

The problem physical domain is denoted by $\Omega$, while the parametric domain is denoted by $\hat{\Omega}$. In $2 \mathrm{D}$, the parametric domain is a rectangle given by $\hat{\Omega}=\Xi \otimes \mathcal{H}$, while in $3 \mathrm{D}$ it is a cuboid given by $\hat{\Omega}=\Xi \otimes \mathcal{H} \otimes \mathcal{Z}$. Both $\hat{\Omega}$ and $\Omega$ are referred to as the patch. While most geometries utilized for academic test cases can be modeled with a single patch, more complicated shapes are typically comprised of multiple patches. The patches are typically merged with $C^{0}$ continuity.

\subsection{Construction of the divergence-conforming B-spline spaces}

We employ divergence-conforming B-splines to approximate the velocity and pressure solutions of the weak form of the Navier-Stokes problem given by Eq. (2). In the divergence-conforming Bspline construction, the velocity and pressure spaces are first defined on the parametric domain $\hat{\Omega}$. Following [29], in 2D, the function spaces are given by

$$
\begin{aligned}
\hat{V}^{h} & =S_{\alpha_{1}, \alpha_{2}-1}^{p, q-1} \times S_{\alpha_{1}-1, \alpha_{2}}^{p-1, q}, \\
\hat{Q}^{h} & =S_{\alpha_{1}-1, \alpha_{2}-1}^{p-1, q-1},
\end{aligned}
$$

while in $3 \mathrm{D}$ their definitions become

$$
\begin{aligned}
\hat{V}^{h} & =S_{\alpha_{1}, \alpha_{2}-1, \alpha_{3}-1}^{p, q-1, r-1} \times S_{\alpha_{1}-1, \alpha_{2}, \alpha_{3}-1}^{p-1, q-1} \times S_{\alpha_{1}-1, \alpha_{2}-1, \alpha_{3}}^{p-1, q-1, r}, \\
\hat{Q}^{h} & =S_{\alpha_{1}-1, \alpha_{2}-1, \alpha_{3}-1}^{p-1, p-1, r-1} .
\end{aligned}
$$

Here, for the $2 \mathrm{D}$ case,

$$
S_{\alpha_{1}, \alpha_{2}}^{p, q}=\operatorname{span}\left\{B_{i, j}^{p, q}(\xi, \eta)\right\}_{i=1, j=1}^{n, m}
$$

while in $3 \mathrm{D}$,

$$
S_{\alpha_{1}, \alpha_{2}, \alpha_{3}}^{p, q, r}=\operatorname{span}\left\{B_{i, j, k}^{p, q, r}(\xi, \eta, \zeta)\right\}_{i=1, j=1, k=1}^{n, m, l},
$$

where $\alpha$ 's denote the integer-valued regularity vectors that contain the degree of B-spline basisfunction continuity at the knots.

It is also assumed that the following boundary conditions and constraints are imposed on the spaces: $\hat{v} \cdot \hat{n}=0$ on $\partial \hat{\Omega} \forall \hat{v} \in \hat{V}^{h}$, where $\hat{n}$ is the unit outward normal vector on $\partial \hat{\Omega}$, and $\int_{\hat{\Omega}} \hat{q}^{h} d \hat{\Omega}=0 \forall \hat{q}^{h} \in \hat{Q}^{h}$. With this construction, one can show that $\forall \hat{q}^{h} \in \hat{Q}^{h}$, if $\hat{v}^{h} \in \hat{V}^{h}$, and

$$
\left(\hat{\nabla} \cdot \hat{v}^{h}, \hat{q}^{h}\right)=0
$$


then $\hat{\nabla} \cdot \hat{v}^{h}=0$ pointwise in $\hat{\Omega}$, where $\hat{\nabla}$ is the gradient operator with respect to the parametric coordinates in $\hat{\Omega}$.

In the physical domain, the discrete velocity and pressure spaces may be defined as

$$
\begin{aligned}
& V^{h}=\left\{v \in H_{0}(\operatorname{div}, \Omega) \mid l_{u}(v) \in \hat{V}^{h}\right\}, \\
& Q^{h}=\left\{q \in L_{0}^{2}(\Omega) \mid l_{p}(q) \in \hat{Q}^{h}\right\},
\end{aligned}
$$

where l's denote the following pullback operations,

$$
\begin{aligned}
& l_{u}(v)=\operatorname{det}(D F) D F^{-1}(v \circ F), \quad v \in H_{0}(\operatorname{div}, \Omega), \\
& l_{p}(q)=q \circ F, \quad q \in L_{0}^{2}(\Omega) .
\end{aligned}
$$

In Eqs. (20)-(21), $F: \hat{\Omega} \rightarrow \Omega$ is the geometrical mapping expressed by Eqs. (9) and (10) for the $2 \mathrm{D}$ and $3 \mathrm{D}$ cases, respectively, and $D F$ is the jacobian of that mapping. We note that the expression given by Eq. (20) is the well-known Piola transformation in continuum mechanics, while Eq. (21) is the standard mapping of a scalar field. With this construction, if the continuity equation in the Navier-Stokes problem is discretized using the Galerkin technique, the resulting discrete velocity is guaranteed to be pointwise divergence-free in the physical domain $\Omega$.

It is convenient to introduce $k$, the polynomial degree of the divergence-conforming B-spline space $V^{h} \times Q^{h}$, defined as $k:=p-1$, where we understand that $p=q=r$ and $\alpha_{i}=p-1$ in Eq. (11)-(14).

Remark The spaces $S_{\alpha_{1}, \alpha_{2}}^{p, q}$ and $S_{\alpha_{1}, \alpha_{2}, \alpha_{3}}^{p, q, r}$ must be comprised of at least $C^{1}$-continuous quadratic splines in order to produce a conforming discretization for the viscous terms in the Navier-Stokes momentum equations. Such smooth discretizations, while straightforward for IGA, present a challenge for standard FEM.

\section{Divergence-conforming VMS}

As a first step in the development of a VMS formulation, orthogonal projectors are defined for the velocity and pressure function spaces as $\mathcal{P}_{u}: V \rightarrow V^{h} \subsetneq V$ and $\mathcal{P}_{p}: Q \rightarrow Q^{h} \subsetneq Q$. Here, $V^{h}$ and $Q^{h}$ are the divergence-conforming B-spline spaces developed in the previous section. We think of $\mathcal{P}_{u}$ as the $H_{0}^{1}$-projector and $\mathcal{P}_{p}$ as the $L^{2}$-projector. These projectors induce an orthogonal decomposition of the velocity and pressure spaces, as well as the corresponding test-function spaces, as $V=V^{h} \oplus V^{\prime}$ and $Q=Q^{h} \oplus Q^{\prime}$, where the objects marked by 'denote spaces of unresolved or fine-scale components of the velocity and pressure fields.

With this decomposition, the weak form given by Eq. (2) splits into two subproblems:

$$
B\left(\left(u^{h}+u^{\prime}, p^{h}+p^{\prime}\right) ;\left(v^{h}, q^{h}\right)\right)=F\left(v^{h}, q^{h}\right) \quad \forall\left(v^{h}, q^{h}\right) \in V^{h} \times Q^{h},
$$


and

$$
B\left(\left(u^{h}+u^{\prime}, p^{h}+p^{\prime}\right) ;\left(v^{\prime}, q^{\prime}\right)\right)=F\left(v^{\prime}, q^{\prime}\right) \quad \forall\left(v^{\prime}, q^{\prime}\right) \in V^{\prime} \times Q^{\prime} .
$$

Here Eqs. (22) and (23) are associate with the coarse- and fine-scale problems, respectively. The main idea of the VMS technique is to express the fine scales in the coarse-scale problem as functions of the resolved velocity and pressure fields. For that purpose, the fine-scale problem is employed to develop such expressions for the fine scales. See [5] for the details of this process in the context the Navier-Stokes equations of incompressible flows.

In this work we deviate from [5], in that we employ the divergence-conforming B-spline construction to develop a VMS formulation that gives a pointwise satisfaction of the incompressibility constraint on the discrete velocity field, that is,

$$
\nabla \cdot u^{h}=0 \text { in } \Omega \times I .
$$

Examining the weak form of the continuity equation, and introducing the above constraint, gives

$$
0=(\nabla \cdot u, q)=\left(\nabla \cdot\left(u^{h}+u^{\prime}\right), q\right)=\left(\nabla \cdot u^{\prime}, q\right) \quad \forall q \in Q
$$

which implies that the the fine scales are also divergence-free (in the sense of $L^{2}$ ). These constraints on the velocity coarse and fine scales were not a part the original VMS formulation in [5], but are explicitly accounted for in the present methodology.

Before stating the divergence-conforming VMS formulation, we make the following observations:

1. The definition of $\mathcal{P}_{u}$ implies $\left(\nabla v^{h}, \nabla u^{\prime}\right)=0 \forall v^{h} \in V^{h}$ and $\forall u^{\prime} \in V^{\prime}$. That is, the velocity coarse and fine scales are orthogonal with respect to the $H_{0}^{1}$ inner product. The same observation was made in [5].

2. Since Eq. (25) holds for all $q \in Q$, it also holds for all $q^{h} \in Q^{h}$, namely

$$
\left(\nabla \cdot u^{\prime}, q^{h}\right)=0 \quad \forall q^{h} \in Q^{h},
$$

which states that the resulting discrete continuity equation is not affected by the velocity fine scales. As a result, in the divergence-conforming VMS formulation, the continuity equation is discretized in the Galerkin form, leading to a pointwise satisfaction of the incompressibility constraint by the discrete velocity field.

3. The definition of the projector $\mathcal{P}_{p}$ may be changed slightly from the usual $L^{2}$ projector to enforce the following orthogonality condition:

$$
\left(l_{p}\left(p^{\prime}\right), \hat{q}^{h}\right)_{\hat{\Omega}}=0 \forall \hat{q}^{h} \in \hat{Q}^{h},
$$


where $(\cdot, \cdot)_{\hat{\Omega}}$ denotes the $L^{2}$ inner product over the parametric domain. The condition given by Eq. (27) states that the pullback of the pressure fine scales is orthogonal to the pressure coarse scales in the parametric domain. Using this definition of the projector, we compute

$$
\begin{aligned}
\left(p^{\prime}, \nabla \cdot v^{h}\right) & =\int_{\hat{\Omega}} l_{p}\left(p^{\prime}\right) \operatorname{det}(D F)\left(\hat{\nabla}\left(v^{h} \circ F\right): D F^{-1}\right) d \hat{\Omega} \\
& =\int_{\hat{\Omega}} l_{p}\left(p^{\prime}\right) \hat{\nabla} \cdot\left(\operatorname{det}(D F) D F^{-1}\left(v^{h} \circ F\right)\right) d \hat{\Omega}=\left(l_{p}\left(p^{\prime}\right), \hat{\nabla} \cdot l_{u}\left(v^{h}\right)\right)_{\hat{\Omega}},
\end{aligned}
$$

where the Piola identity was used to go from the first to second line. Recognizing that $\hat{\nabla} \cdot l_{u}\left(v^{h}\right) \in \hat{Q}^{h}$, and using the orthogonality condition given by Eq. (27), we conclude that

$$
\left(p^{\prime}, \nabla \cdot v^{h}\right)=0 \forall v^{h} \in V^{h},
$$

which implies that the coarse-scale momentum equations are not affected by $p^{\prime}$ and obviates the need to model the pressure fine scales.

The above observations, combined with the coarse-scale equations Eq. (22), lead to the following divergence-conforming VMS formulation: Find $u^{h} \in V^{h}$ and $p^{h} \in Q^{h}$, such that $\forall v^{h} \in V^{h}$ and $q^{h} \in Q^{h}$,

$$
\begin{aligned}
B_{D C V M S}\left(\left(u^{h}, p^{h}\right) ;\left(v^{h}, q^{h}\right)\right) & =F_{D C V M S}\left(v^{h}, q^{h}\right), \\
B_{D C V M S}\left(\left(u^{h}, p^{h}\right) ;\left(v^{h}, q^{h}\right)\right) & =B\left(\left(u^{h}, p^{h}\right) ;\left(v^{h}, q^{h}\right)\right)-\left(u^{h} \otimes u^{\prime}, \nabla v^{h}\right) \\
& -\left(u^{\prime} \otimes u^{h}, \nabla v^{h}\right)-\left(u^{\prime} \otimes u^{\prime}, \nabla v^{h}\right), \\
F_{D C V M S}\left(v^{h}, q^{h}\right) & =F\left(v^{h}, q^{h}\right),
\end{aligned}
$$

where the velocity fine scales $u^{\prime}$ are understood as functions of the resolved velocity and pressure fields. Expressions for $u^{\prime}$ are provided in the following section.

\section{The fine-scale problem}

The fine-scale problem given by Eq. (23) may be written as: Find $u^{\prime} \in V^{\prime}$ and $p^{\prime} \in Q^{\prime}$, such that $\forall v^{\prime} \in V^{\prime}$ and $q^{\prime} \in Q^{\prime}$,

$$
\begin{aligned}
<\mathcal{L}_{u} u^{\prime}, v^{\prime}>- & \left(p^{\prime}, \nabla \cdot v^{\prime}\right)=-<r\left(u^{h}, p^{h}\right), v^{\prime}> \\
\left(\nabla \cdot u^{\prime}, q^{\prime}\right) & =0,
\end{aligned}
$$

where $r(u, p)=\partial_{t} u+u \cdot \nabla u+\nabla p-\nu \Delta u-f$ is the strong residual of the Navier-Stokes momentum equations, $\mathcal{L}_{u}(\cdot)=\partial_{t}(\cdot)+u \cdot \nabla(\cdot)-\nu \Delta(\cdot)$ is the convection-diffusion operator, and $\langle\cdot, \cdot\rangle$ denotes the appropriate duality pairing. 
We note that the formulation given by Eq. (33) is posed over a space of fine scales, characterized by a constraint $\mathcal{P}_{u} u^{\prime}=0$. Following the developments of [37], the formulation given by Eq. (33) may be posed over an unconstrained space by using the Lagrange multiplier approach, namely: Find $u^{\prime} \in V, p^{\prime} \in Q$, and $\bar{\lambda} \in V^{h *}$, the dual of $V^{h}$, such that $\forall v \in V, q \in Q$, and $\bar{\mu} \in V^{h *}$

$$
\begin{aligned}
<\mathcal{L}_{u} u^{\prime}, v>+<\mathcal{P}_{u}^{T} \bar{\lambda}, v>-\left(p^{\prime}, \nabla \cdot v\right) & =-<r\left(u^{h}, p^{h}\right), v>, \\
<\mathcal{P}_{u} u^{\prime}, \bar{\mu}> & =0, \\
\left(\nabla \cdot u^{\prime}, q\right) & =0,
\end{aligned}
$$

where we also used the fact that $\left(p^{\prime}, \nabla \cdot v^{\prime}\right)=\left(p^{\prime}, \nabla \cdot v\right)$ due to the pressure projector.

We can formally state the solution to the fine-scale problem given by Eq. (34) in terms of the coarse-scale residual and fine-scale pressure gradient as

$$
u^{\prime}=\mathcal{G}^{\prime}\left(-r\left(u^{h}, p^{h}\right)-\nabla p^{\prime}\right),
$$

where $\mathcal{G}^{\prime}$ is the fine-scale Green's function for the convection-diffusion operator $\mathcal{L}_{u}$. This object was derived in [37] and given by

$$
\mathcal{G}^{\prime}=\mathcal{G}-\mathcal{G} \mathcal{P}_{u}^{T}\left(\mathcal{P}_{u} \mathcal{G} \mathcal{P}_{u}^{T}\right)^{-1} \mathcal{P}_{u} \mathcal{G}
$$

with $\mathcal{G}$ being the full Green's function for the convection-diffusion operator.

In [37] the authors showed that while both $\mathcal{G}$ and $\mathcal{G}^{\prime}$ are global operators, $\mathcal{G}^{\prime}$ is much more localized than $\mathcal{G}$. This fact was used as a justification for stabilized methods, which approximate the action of $\mathcal{G}^{\prime}$ by a multiplication with a scalar-valued function $\tau$, also known as the stabilization parameter. We follow the same reasoning here and replace $\mathcal{G}^{\prime}$ in Eq. (35) to arrive at

$$
\begin{aligned}
\tau^{-1} u^{\prime}+\nabla p^{\prime} & =-r\left(u^{h}, p^{h}\right), \\
\nabla \cdot u^{\prime} & =0,
\end{aligned}
$$

which yields a Darcy problem with diffusivity $\tau^{-1}$ driven by the momentum residual of the coarsescale solution. The parameter $\tau$ employed in this work is given by

$$
\tau=\left(4 / \delta t^{2}+u \cdot G u+C_{I} \nu^{2}|G|^{2}\right)^{-1 / 2},
$$

where $\delta t$ is the time step, $G=\hat{D F}{ }^{-T} \hat{D F} F^{-1}, \hat{D F}$ the jacobian of the mapping from a bi-unit square (in $2 \mathrm{D}$ ) or cube (in 3D) to the physical element, and $C_{l}$ is a positive constant arising in the element inverse estimate. This definition is taken directly from [5]. For other definitions of stabilization parameters the reader is referred to $[46,45,47,33]$.

In the discrete setting, we approximate the solution of Eq. (37) using the Galerkin method and divergence-conforming B-spline discretization for the velocity and pressure restricted to each individual element. We consider the following alternatives for the fine-scale problem: 
1. The Darcy-Dirichlet problem: Find $u^{\prime} \in V_{0}^{h}\left(\Omega_{e}\right)$ and $p^{\prime} \in Q_{0}^{h}\left(\Omega_{e}\right)$, such that $\forall v^{\prime} \in V_{0}^{h}\left(\Omega_{e}\right)$ and $q^{\prime} \in Q_{0}^{h}\left(\Omega_{e}\right)$,

$$
\begin{aligned}
\left(\tau^{-1} u^{\prime}, v^{\prime}\right)_{e}- & \left(p^{\prime}, \nabla \cdot v^{\prime}\right)_{e}=-\left(r\left(u^{h}, p^{h}\right), v^{\prime}\right)_{e} \\
\left(\nabla \cdot u^{\prime}, q^{\prime}\right)_{e} & =0
\end{aligned}
$$

where $\Omega_{e}$ is the element domain, $(\cdot, \cdot)_{e}$ is the $L^{2}$ inner product over $\Omega_{e}, V_{0}^{h}\left(\Omega_{e}\right)$ is the divergence-conforming spline space for the velocity restricted to $\Omega_{e}$ with boundary conditions $l_{u}\left(u^{\prime}\right) \cdot \hat{n}=0$ on $\partial \hat{\Omega}_{e}$, and $Q_{0}^{h}\left(\Omega_{e}\right)$ the divergence-conforming spline space for the pressure restricted to $\Omega_{e}$ and satisfying the zero-mean condition $\left(p^{\prime}, 1\right)_{e}=0$.

2. The Darcy-Neumann problem. Here we relax the constraints on the velocity fine scales at the element boundary, and formulate the discrete fine-scale problem as follows: Find $u^{\prime} \in V^{h}\left(\Omega_{e}\right)$ and $p^{\prime} \in Q^{h}\left(\Omega_{e}\right)$, such that $\forall v^{\prime} \in V^{h}\left(\Omega_{e}\right)$ and $q^{\prime} \in Q^{h}\left(\Omega_{e}\right)$,

$$
\begin{aligned}
\left(\tau^{-1} u^{\prime}, v^{\prime}\right)_{e}- & \left(p^{\prime}, \nabla \cdot v^{\prime}\right)_{e}=-\left(r\left(u^{h}, p^{h}\right), v^{\prime}\right)_{e} \\
\left(\nabla \cdot u^{\prime}, q^{\prime}\right)_{e} & =0 .
\end{aligned}
$$

The above variational form implies that, in a weak sense, $p^{\prime}=0$ on $\partial \Omega_{e}$.

3. The Darcy-Periodic problem. In this final approach, we enforce periodic boundary conditions on both the velocity and pressure fine scales at the element boundary. This approach is inspired by Kolmogorov's hypothesis of local homogeneity, which states that at sufficiently high Reynolds numbers, the small-scale turbulent motions associated with the inertial subrange and dissipation range are statistically homogeneous. While we do not explore this approach further within the current work, we will examine it in detail in the future.

The complete divergence-conforming VMS formulation is obtained by inserting the resulting $u^{\prime}$ satisfying the discrete Darcy-Dirichlet, Darcy-Neumann, or Darcy-Periodic problems into the formulation given by Eq. (30). It is important to note that since a divergence-conforming discretization of the Darcy problem is employed at the element level, the resulting velocity fine scales are, as desired, pointwise divergence-free.

Remark The Darcy formulation may be thought of as the element-wise constrained minimization problem:

$$
\min _{u^{\prime} \in V^{h}\left(\Omega_{e}\right)} \max _{p^{\prime} \in Q^{h}\left(\Omega_{e}\right)} \frac{1}{2}\left\|u^{\prime}+\tau r\left(u^{h}, p^{h}\right)\right\|_{L^{2}\left(\Omega_{e}\right), \tau^{-1}}^{2}-\left(p^{\prime}, \nabla \cdot u^{\prime}\right)_{e},
$$

where $\|f\|_{L^{2}\left(\Omega_{e}\right), \tau^{-1}}=\left(f, \tau^{-1} f\right)_{e}^{1 / 2}$ is the weighted $L^{2}\left(\Omega_{e}\right)$ norm. In this spirit, other norms may be considered for the minimization problem. 
Remark The velocity field is completely decoupled from the pressure field in the Darcy-Dirichlet problem. This fact may be observed by strongly enforcing the divergence-free constraint within the fine-scale velocity trial space, resulting in the following simplified problem: Find $u^{\prime} \in V_{0}^{h}\left(\Omega_{e}\right)$ such that $\forall v^{\prime} \in \stackrel{\circ}{V}_{0}^{h}\left(\Omega_{e}\right)$,

$$
\left(\tau^{-1} u^{\prime}, v^{\prime}\right)_{e}=-\left(\mathcal{L}_{u^{h}} u^{h}-f, v^{\prime}\right)_{e}
$$

where

$$
\stackrel{\circ}{V}_{0}^{h}\left(\Omega_{e}\right):=\left\{\mathbf{v}_{h} \in V_{0}^{h}\left(\Omega_{e}\right): \nabla \cdot \mathbf{v}_{h}=0\right\} .
$$

The decoupling of the velocity and pressure fields in the Darcy-Dirichlet problem allows for the use of efficient linear solvers which act purely on the divergence-free velocity space. It should be noted that the velocity field is not completely decoupled from the pressure field in the Darcy-Neumann and Darcy-Periodic problems.

Remark Instead of making a simple estimate $\mathcal{G}^{\prime} \approx \tau$, one could attempt to compute an approximate solution of the linearized fine-scale problem as: Find $u^{\prime} \in \bar{V}_{0}\left(\Omega_{e}\right)$ and $p^{\prime} \in \bar{Q}_{0}\left(\Omega_{e}\right)$, such that $\forall v^{\prime} \in \bar{V}_{0}\left(\Omega_{e}\right)$ and $q^{\prime} \in \bar{Q}_{0}\left(\Omega_{e}\right)$,

$$
\begin{aligned}
<\mathcal{L}_{u} u^{\prime}, v^{\prime}>-\left(p^{\prime}, \nabla \cdot v^{\prime}\right) & =-<r\left(u^{h}, p^{h}\right), v^{\prime}>, \\
\left(\nabla \cdot u^{\prime}, q^{\prime}\right) & =0 .
\end{aligned}
$$

This approach is essentially a divergence-conforming extension of the residual-free bubble technique (see [14, 13]), which requires well-designed discrete approximation spaces for the velocity and pressure (denoted here by $\bar{V}_{0}\left(\Omega_{e}\right)$ and $\bar{Q}_{0}\left(\Omega_{e}\right)$, respectively) in order to guarantee accuracy and stability for a large range of Reynolds numbers. For example, element-level polynomial spaces are inefficient in capturing the fine-scale behavior in the advection-dominated regime (see, e.g., [37]). As a result, a more sophisticated approach is required to construct the fine-scale spaces, which now has the additional challenge of satisfying the fine-scale-velocity zero-divergence constraint.

Remark In the case the divergence-free constraint on the fine scales is relaxed, and the approximations $u^{\prime}=-\tau r\left(u^{h}, p^{h}\right)$ and $p^{\prime}=-\tau_{C} \nabla \cdot u^{h}$ are adopted for the fine scales, one obtains the residual-based variational multiscale (RBVMS) formulation from [5]: Find $u^{h} \in \bar{V}$ and $p^{h} \in \bar{Q}$, such that $\forall v^{h} \in \bar{V}$ and $q^{h} \in \bar{Q}$,

$$
\begin{aligned}
B_{V M S}\left(\left(u^{h}, p^{h}\right),\left(v^{h}, q^{h}\right)\right) & =F_{V M S}\left(v^{h}, q^{h}\right), \\
B_{V M S}\left(\left(u^{h}, p^{h}\right),\left(v^{h}, q^{h}\right)\right) & =B\left(\left(u^{h}, p^{h}\right),\left(v^{h}, q^{h}\right)\right)+\left(u^{h} \otimes \tau r\left(u^{h}, p^{h}\right), \nabla v^{h}\right) \\
& +\left(\tau r\left(u^{h}, p^{h}\right) \otimes u^{h}, \nabla v^{h}\right)-\left(\tau r\left(u^{h}, p^{h}\right) \otimes \tau r\left(u^{h}, p^{h}\right), \nabla v^{h}\right), \\
& +\left(\tau_{C} \nabla \cdot u^{h}, \nabla \cdot v^{h}\right)+\left(\tau r\left(u^{h}, p^{h}\right), \nabla q^{h}\right), \\
F_{V M S}\left(v^{h}, q^{h}\right) & =F\left(v^{h}, q^{h}\right) .
\end{aligned}
$$


The RBVMS is typically employed in the context of equal-order velocity-pressure discretizations, hence a slightly different notation for the discrete spaces, $\bar{V}$ and $\bar{Q}$, is employed for this case.

Remark The classical SUPG/PSPG formulation [48] is obtained by neglecting the pressure fine scales, as well as terms identified with the Reynolds and cross stresses in the terminology of classical LES and RANS turbulence modeling [49, 43]: Find $u^{h} \in \bar{V}$ and $p^{h} \in \bar{Q}$, such that $\forall v^{h} \in \bar{V}$ and $q^{h} \in \bar{Q}$,

$$
\begin{aligned}
B_{S U P S}\left(\left(u^{h}, p^{h}\right),\left(v^{h}, q^{h}\right)\right) & =F_{S U P S}\left(v^{h}, q^{h}\right), \\
B_{S U P S}\left(\left(u^{h}, p^{h}\right),\left(v^{h}, q^{h}\right)\right) & =B\left(\left(u^{h}, p^{h}\right),\left(v^{h}, q^{h}\right)\right) \\
& +\left(r\left(u^{h}, p^{h}\right), \tau u^{h} \cdot \nabla v^{h}\right)+\left(r\left(u^{h}, p^{h}\right), \tau \nabla q^{h}\right), \\
F_{S U P S}\left(v^{h}, q^{h}\right) & =F\left(v^{h}, q^{h}\right) .
\end{aligned}
$$

The SUPG/PSPG formulation is also typically employed for equal-order velocity-pressure discretizations.

Remark The classical SUPG formulation, as presented in its original form in [15], may be obtained by neglecting the residual-based terms in the continuity equation: Find $u^{h} \in V^{h}$ and $p^{h} \in Q^{h}$, such that $\forall v^{h} \in V^{h}$ and $q^{h} \in Q^{h}$,

$$
\begin{aligned}
B_{S U P G}\left(\left(u^{h}, p^{h}\right),\left(v^{h}, q^{h}\right)\right) & =F_{S U P G}\left(v^{h}, q^{h}\right), \\
B_{S U P G}\left(\left(u^{h}, p^{h}\right),\left(v^{h}, q^{h}\right)\right) & =B\left(\left(u^{h}, p^{h}\right),\left(v^{h}, q^{h}\right)\right)+\left(r\left(u^{h}, p^{h}\right), \tau u^{h} \cdot \nabla v^{h}\right), \\
F_{S U P G}\left(v^{h}, q^{h}\right) & =F\left(v^{h}, q^{h}\right) .
\end{aligned}
$$

In the case of SUPG, while the residual-based terms provide the necessary convective stabilization, one still needs to employ velocity-pressure discretizations that satisfy the Babuška-Brezzi (BB) condition $[3,12]$ in order to have a stable and optimally convergent method. The framework of divergence-conforming B-splines is well suited for this purpose since, by construction, it produces BB-stable velocity-pressure pairs.

\section{Boundary and patch-interface conditions}

In the context of divergence-conforming B-splines, imposition of essential boundary conditions, as well as enforcement of kinematics and traction continuity across patches in the case of multipatch B-spline discretizations, deserves some attention. As advocated in [29], the most natural framework for boundary- and patch-interface-condition imposition involves strong enforcement of no-penetration and weak enforcement of no-slip conditions. Nitsche's approach [42], later extended to weak-essential-boundary-condition [6] and sliding-interface $[7,32]$ formulations, is employed here 
for that purpose in the form described in [29]. The following weak-boundary-condition and patchinterface terms are added to any of the discrete formulations discussed previously:

$$
\begin{aligned}
& +\sum_{\partial e \in \mathcal{F}^{h}}\left(-\left(t\left(u^{h}\right), v_{\tau}^{h}\right)_{\partial e}-\left(u_{\tau}^{h}, t\left(v^{h}\right)\right)_{\partial e}+\frac{C_{B} \nu}{h_{\partial e}}\left(u_{\tau}^{h}, v_{\tau}^{h}\right)_{\partial e}\right) \\
& \quad+\sum_{\partial e \in \mathcal{I}^{h}}\left(-\left(\left\{t\left(u^{h}\right)\right\}, \llbracket v_{\tau}^{h} \rrbracket\right)_{\partial e}-\left(\llbracket u_{\tau}^{h} \rrbracket,\left\{\left\{t\left(v^{h}\right)\right\}\right)_{\partial e}+\frac{C_{B} \nu}{h_{\partial e}}\left(\llbracket u_{\tau}^{h} \rrbracket, \llbracket v_{\tau}^{h} \rrbracket\right)_{\partial e}\right) .\right.
\end{aligned}
$$

Here, $(\cdot)_{\tau}=(\cdot)-[(\cdot) \cdot n] n$ is the vector tangential component, $t(\cdot)=2 \nu \nabla^{s}(\cdot) n$ is the viscous traction operator, $\llbracket \cdot \rrbracket$ and $\left\{[\cdot\}\right.$ denote the usual jump and average operators, $\partial e$ is the element boundary, $h_{\partial e}$ is the local mesh size, $C_{B}>0$ is the nondimensional boundary stabilization (or penalty) parameter, and $\mathcal{I}^{h}$ and $\mathcal{F}^{h}$ denote the sets of patch-interface elements and boundary elements, respectively.

Remark We note that near solid surfaces, weak enforcement of no-slip boundary conditions may be interpreted as near-wall modeling in boundary-layer turbulent flows (see $[8,30]$ for details). In addition, because residual-based VMS was developed and validated as a turbulence model applicable in the LES regime, the present formulation, in the terminology of turbulence modeling, may be though of as divergence-conforming LES with near-wall modeling.

\section{Numerical examples}

The divergence-conforming VMS technique is tested using two 2D laminar-flow benchmark problems, a test case involving Taylor-Green vortex flow at Reynolds number $R e=1600$, and a test case involving turbulent channel flow at friction-velocity-based Reynolds number $R e_{\tau}=395$. In the case of $2 \mathrm{D}$ benchmarks, the divergence-conforming VMS technique is compared to Galerkin and SUPG formulations, also discretized using divergence-conforming B-splines. In the case of the TaylorGreen vortex flow, the divergence-conforming VMS technique is compared to the Galerkin formulation, also discretized using divergence-conforming B-splines. The version of divergence-conforming VMS tested in this context corresponds to the Darcy-Dirichlet problem given by Eq. (39). In the case of turbulent channel flow, the standard and divergence-conforming VMS formulations are compared, where the divergence-conforming version makes use of the Darcy-Neumann formulation given by Eq. (40).

\subsection{Regularized lid-driven cavity}

We consider a steady, regularized lid-driven cavity flow in a unit square with a body force. The analytical solution for this case is known [44], and is given by

$$
\begin{aligned}
& u=8\left\{f_{1} f_{2}^{\prime},-f_{1}^{\prime} f_{2}\right\}, \\
& p=8 \nu\left(F_{1} f_{2}^{\prime \prime \prime}+f_{1}^{\prime} f_{2}^{\prime}\right)+32 f_{1}^{2}\left(f_{2} f_{2}^{\prime \prime}-f_{2}^{\prime 2}\right),
\end{aligned}
$$



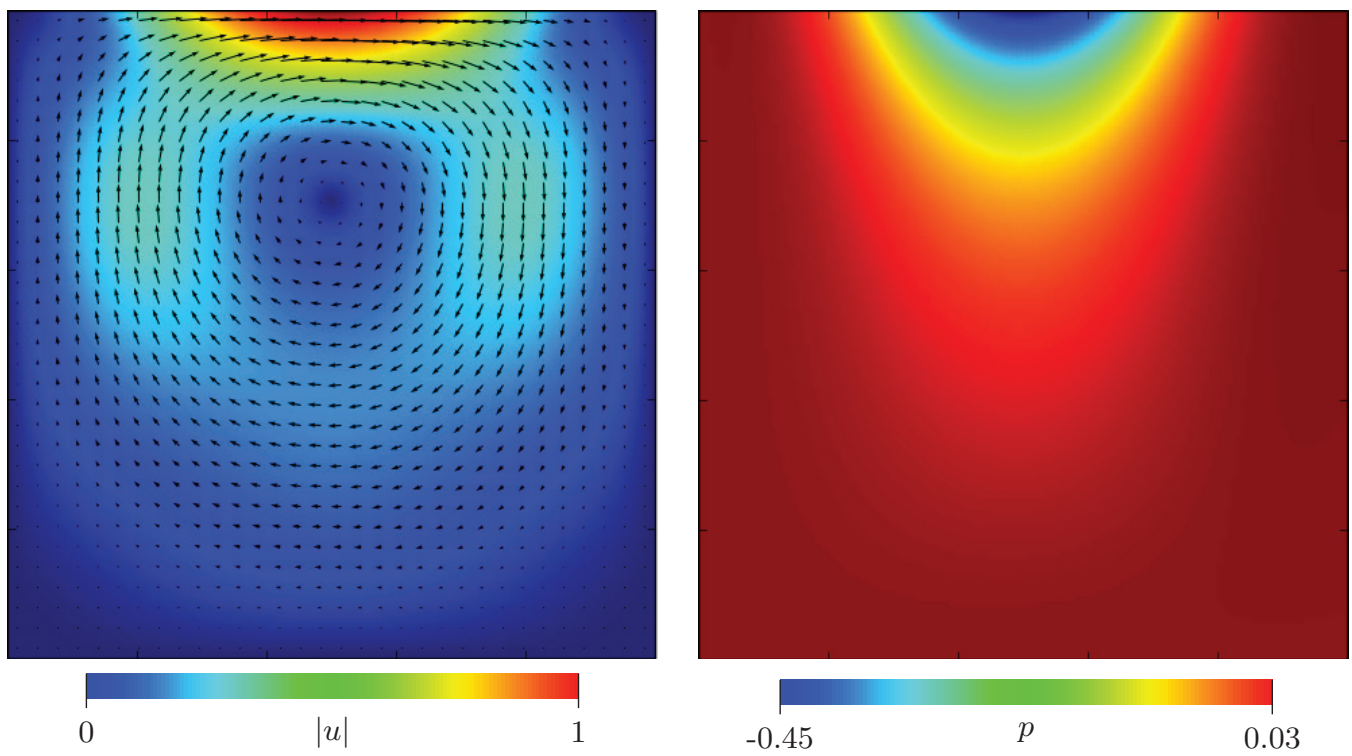

Figure 1: Velocity and pressure for the regularized cavity problem at $R e=1000$.

where $(\cdot)^{\prime}$ denotes the derivative and $F_{1}^{\prime}=f_{1}=x^{4}-2 x^{3}+x^{2}$ and $f_{2}=y^{4}-y^{2}$. Note that $\|u\|_{\infty}=|u(1 / 2,1)|=1$, so the Reynolds number is $\operatorname{Re}=1 / \nu$. We compute the solution on a family of uniform meshes $1 / h \in\{8,16,32,64\}$ for $R e=1000$, using the Reynolds-number continuation technique with levels $\operatorname{Re} \in\{100,400,1000\}$. The value of the boundary-stabilization parameter is set to $C_{B}=5(k+1)$.

A mesh convergence study is performed for different polynomial orders to compare the Galerkin and SUPG methods with the newly proposed VMS method, where the fine-scales are approximated with element-wise Darcy-Dirichlet and Darcy-Neumann problems (where we choose $k=1$ for the fine-scale problems), see Figure 2. The convergences rates are also summarized in Table 1. It is observed that optimal convergence rates are attained in all cases. The slightly higher rates reported in the Darcy-Neumann case show that the asymptotic range starts later compared to the other models. This phenomenon is under current investigation. The consistency of the technique is verified by performing the computation with splines of order eight, which captures the velocity and pressure fields exactly, producing only machine-precision error (see Table 1.) This example also shows that in the case of SUPG and VMS the stabilizing residual-based terms vanish sufficiently quickly with mesh refinement to guarantee optimal convergence of these techniques. 


\begin{tabular}{c|c|c|c|c|c|c|c|c}
\multirow{2}{*}{$k$} & \multicolumn{4}{|c|}{$\left\|u-u^{h}\right\|_{H_{0}^{1}(\Omega)}$} & \multicolumn{4}{|c}{$\left\|p-p^{h}\right\|_{L^{2}(\Omega)}$} \\
\cline { 2 - 9 } & Gal. & SUPG & DD & DN & Gal. & SUPG & DD & DN \\
\hline 1 & 1.0 & 1.0 & 1.0 & 1.3 & 2.0 & 2.1 & 2.0 & 2.4 \\
2 & 2.0 & 2.0 & 2.0 & 2.3 & 3.2 & 3.2 & 3.2 & 2.9 \\
8 & - & - & - & - & - & - & - & -
\end{tabular}

Table 1: Error convergence rates for the regularized lid-driven cavity problem at $R e=1000$. A dash indicates that the numerical approximation agrees with the exact solution up to machine precision. Here $k$ denotes the polynomial order of the approximation space, cf. $\S 3.2$.
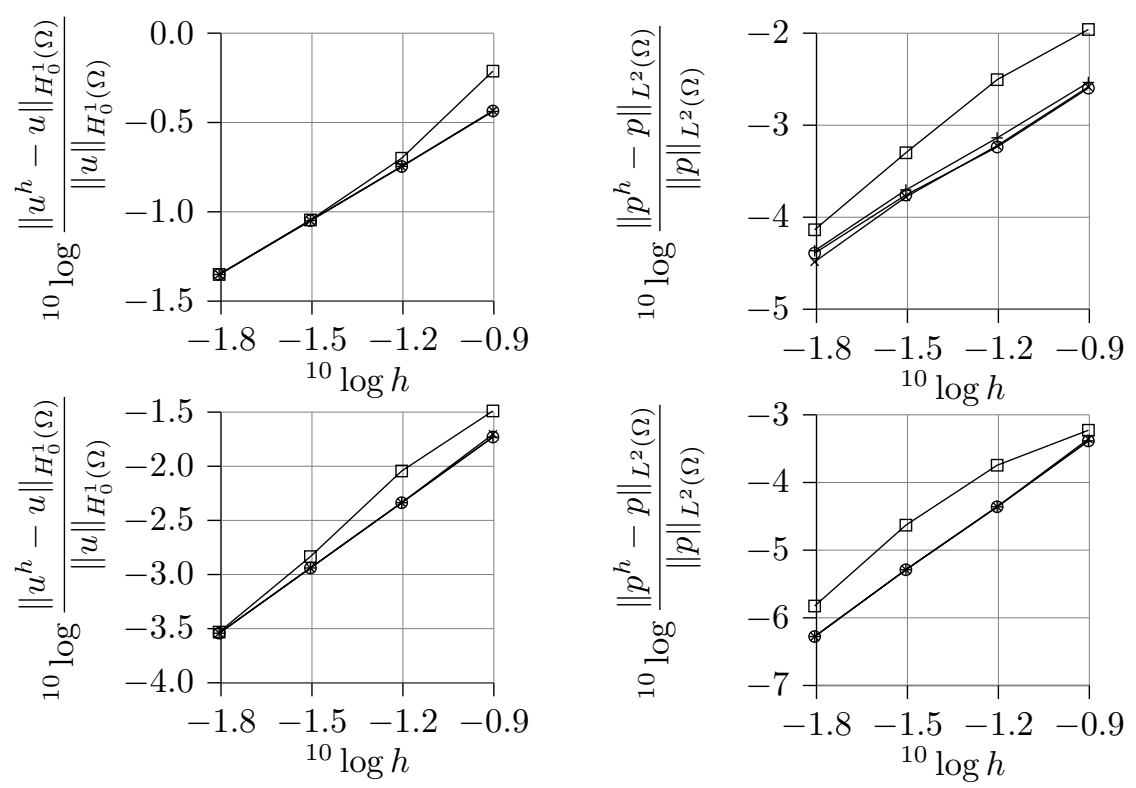

Figure 2: Regularized lid-driven cavity at $\mathrm{Re}=1000$. Velocity (left panels) and pressure (right panels) error convergence under mesh refinement for $k=1$ (top panels) and $k=2$ (bottom panels). Galerkin $(+)$, SUPG $(\times)$, Darcy-Dirichlet $(\bigcirc)$, and Darcy-Neumann $(\square)$ formulations are compared. 


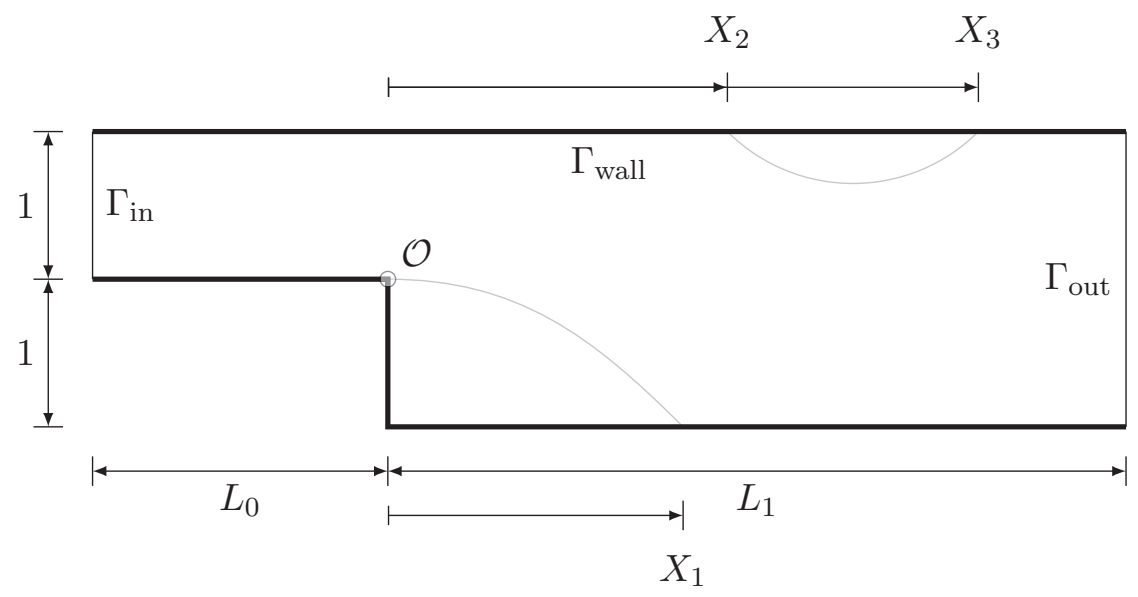

Figure 3: Schematic of the backward-facing step problem.

\subsection{Backward-facing step}

We compute the steady backward-facing step problem, which has been the subject of many investigations and presents a good benchmark for methods verification and comparison due to the availability of well-documented numerical solutions. This is also an example where a multiple-patch discretization is tested. The flow setup is shown in Figure 3, where $\mathcal{O}$ denotes the origin. The boundary conditions are specified as $u=\{6 y(1-y), 0\}$ on $\Gamma$ in, $u=0$ on $\Gamma$ wall, and $-p n+2 \nu \nabla^{s} u \cdot n=0$ on $\Gamma$ out.

Investigations have been performed on the influence of the lengths $L_{0}$ and $L_{1}$ (see Figure 3) and the choice of outflow conditions. It was shown in [22] that the choice $L_{0}=0$ affects the flow solution at moderate Reynolds number. (The Reynolds number in this problem is based on the step height and average inflow velocity (both unity), and the viscosity.) At the same moderate Reynolds number, the flow eventually develops into a Poiseuille profile downstream of the step, and the adequacy of $L_{1}$ is judged by the deviation of the velocity profile from the Poiseuille solution, as proposed in [40]. We note that the flow is not expected to follow the Poiseuille solution at the outflow boundary due to the use of the symmetric-stress boundary conditions, so we examine the velocity profile approximately eight step heights upstream of the outflow. We use grid stretching for higher wall-normal resolution at the downstream horizontal walls, and lower streamwise resolution towards the outflow. We use $L_{0}=5, L_{1}=60$, and $5 \%$ grid stretching. The element size in the vicinity of the step was chosen as $1 / 16$, resulting in a mesh with 21,200 elements. Furthermore, the divergence-conforming approximation space with polynomial degree $k=1$ (according to the definition of §3.2) was used. The singularity at the reentrant corner is not treated explicitly, other than through the discontinuities introduced in to the multi-patch approach required to represent the 

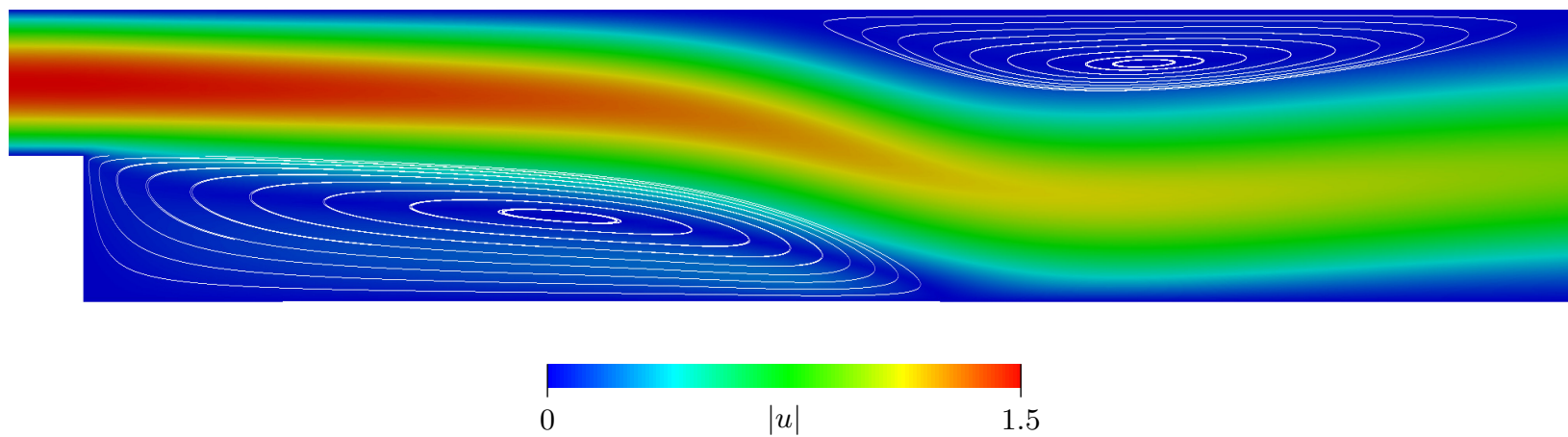

Figure 4: Velocity contours and streamlines for the backward-facing step problem for $\operatorname{Re}=800$. Zoom on the region $[-1,14] \times[-1,1]$ is shown.

geometry. In the case a mesh refinement study is performed, one would needs to employ adaptive refinement near the reentrant corner to recover optimal convergence rates.

To compute the flow separation and reattachment points, we reconstruct the stream function from the velocity solution. Level sets of the stream function correspond to the flow streamlines. In particular, recirculation bubbles are bounded by level sets which include a wall boundary (i.e., a contiguous segment of $\partial \Omega$ where $g \cdot n$ vanishes). Because the discrete velocity solution is pointwise divergence-free, the stream-function reconstruction is exact, which presents an added benefit of the proposed approach. Details of the stream-function reconstruction are provided in the Appendix.

Remark When the boundary-layer mesh is relatively coarse, weak enforcement of boundary conditions leads to nonzero slip velocity at the boundary, which results in some inaccuracy of the streamfunction reconstruction in one layer of elements adjacent to the wall. Therefore, a quadratic fit is employed to recover the separation-bubble locations. This quadratic fit uses level set locations at the first two mesh points away from the boundary, and the fact that the normal gradient of the stream function should vanish at the boundary due to no-slip and no-penetration boundary conditions.

We compute two cases corresponding to $\mathrm{Re}=400$ and $\mathrm{Re}=800$, comparing as before the Galerkin, SUPG and VMS formulations, the latter with a Darcy-Dirichlet fine scale problem. Figure 4 shows the velocity contours and streamlines for the $R e=800$ case. The flow solution is smooth even at patch boundaries. The separation-bubble locations, denoted by $X_{1}, X_{2}$, and $X_{3}$ in Figure 3 , are compared with those reported in [25] in Tables 2 and 3. Good agreement is achieved in all cases, despite the coarse resolution of the approximation space. 


\begin{tabular}{l|rrr} 
& $X_{1}$ & $X_{2}$ & $X_{3}$ \\
\hline$[25]$ & 8.237 & 7.731 & 10.037 \\
\hline Galerkin & 8.305 & 7.244 & 10.398 \\
& $(0.8 \%)$ & $(6.3 \%)$ & $(3.6 \%)$ \\
\hline SUPG & 8.263 & 7.295 & 9.808 \\
& $(0.3 \%)$ & $(5.6 \%)$ & $(2.3 \%)$ \\
\hline Darcy-Dirichlet & 8.305 & 7.242 & 10.401 \\
& $(0.8 \%)$ & $(6.3 \%)$ & $(3.6 \%)$
\end{tabular}

Table 2: Recirculation-bubble locations for $\mathrm{Re}=400$. Comparison with data from [25] is provided in the table.

\begin{tabular}{l|rrr} 
& $X_{1}$ & $X_{2}$ & $X_{3}$ \\
\hline$[25]$ & 11.834 & 9.476 & 20.553 \\
\hline Galerkin & 11.910 & 9.210 & 20.973 \\
& $(0.6 \%)$ & $(2.8 \%)$ & $(2.0 \%)$ \\
\hline SUPG & 11.618 & 8.929 & 21.037 \\
& $(1.8 \%)$ & $(5.8 \%)$ & $(2.4 \%)$ \\
\hline Darcy-Dirichlet & 11.907 & 9.207 & 20.973 \\
& $(0.6 \%)$ & $(2.8 \%)$ & $(2.0 \%)$
\end{tabular}

Table 3: Recirculation-bubble locations for $\mathrm{Re}=800$. Comparison with data from [25] is provided in the table. 


\subsection{Taylor-Green vortex flow at $\mathrm{Re}=1600$}

Three-dimensional Taylor-Green vortex flow is one of the simplest systems in which to study the generation of small scales through vortex stretching and the energy dissipation from the resulting turbulence. The initial condition of Taylor-Green vortex flow is laminar and consists of purely two-dimensional streamlines, but for all time $t>0$, the flow is three-dimensional. As the solution is evolved in time, vortex stretching causes the generation of small-scale motion and eventual transition into turbulence. The initial conditions for this flow are

$$
u_{0}(x, y, z)=\left[\begin{array}{c}
\sin (x) \cos (y) \cos (z) \\
-\cos (x) \sin (y) \cos (z) \\
0
\end{array}\right] .
$$

We simulate Taylor-Green vortex flow at a Reynolds number based on the integral length scale of $\operatorname{Re}=1600$. For this type of flow, this Reynolds number can be defined as $R e=\frac{1}{\nu}$. The flow is periodic in all three spatial directions in the domain $\Omega=(0,2 \pi)^{3}$ and, due to inherent symmetries in the flow, we model the flow using a restricted computational domain of ${ }^{h}=(0, \pi)^{3}$ with impermeable stress-free boundaries. The solution is evolved in time using a semi-implicit method wherein the Crank-Nicolson method is employed to discretize viscous terms while the AdamsBashforth multi-step method is employed to discretize the nonlinear convective terms as well as couple the coarse-scale and fine-scale solutions. A time-step size of $\Delta t=0.0125 h$ is employed in all simulations.

Two simulations of this flow are performed, using: 1) The Galerkin method discretized using divergence-conforming B-splines, and 2) The newly proposed divergence-conforming VMS formulation with the fine scales approximated by the element-level discrete Darcy-Dirichlet problem given by Eq. (39). Divergence-conforming splines of degree $k=1$ are used. For both simulations, a highly unresolved uniform mesh of $32^{3}$ elements is employed. The eddies associated with the length scale set by the mesh size belong to the inertial subrange as required by classical LES techniques.

The time histories of the kinetic energy dissipation rate up to a non-dimensional time of $t=10$ for both simulations are compared to the Fourier-based DNS results of [11] in Figure 5. The Galerkin method over-predicts the energy dissipation rate at all times. In contrast, the VMS formulation provides a much closer agreement to the DNS data, despite the fact that the turbulent flow is highly unresolved. The magnitude of peak dissipation associated with the VMS formulation is much closer to the DNS data than the peak dissipation associated with the Galerkin method, though the time of peak dissipation is under-predicted in both simulations. We remark that the time of peak dissipation might be better predicted with a higher-order fine-scale model. In the current simulation, the degree of the fine-scale model is only chosen to be $k=1$, and degree elevation studies are underway to examine the effect of subgrid model refinement. The energy spectra as a function of wavenumber at $t=10$ are presented in Figure 6 . A reference line with slope $-\frac{5}{3}$ is also plotted. The spectra for both the Galerkin and VMS formulations show the expected behavior, and here the VMS model does not show any appreciable effect on the energy spectrum. 


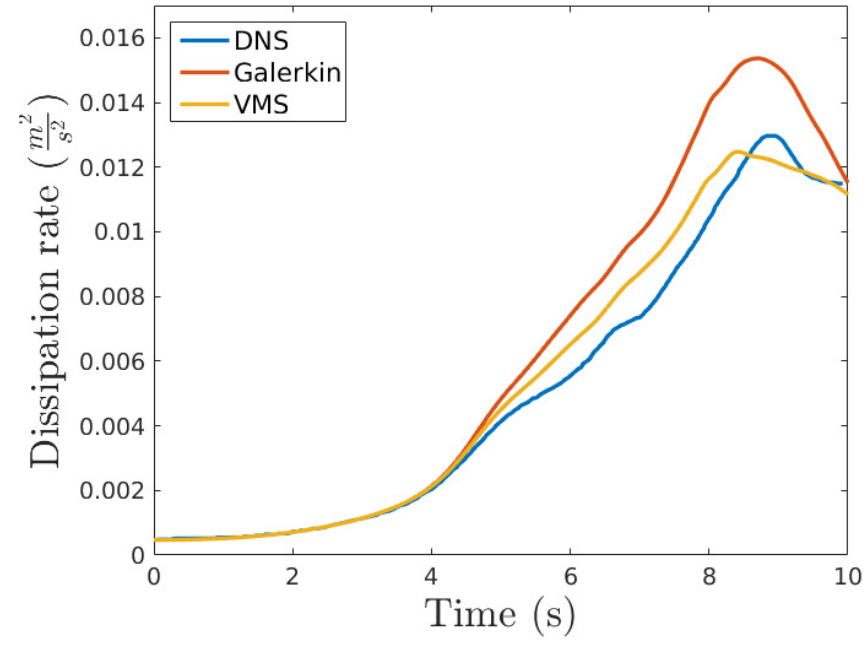

Figure 5: Taylor-Green vortex flow at $\mathrm{Re}=1600$. Dissipation rate.

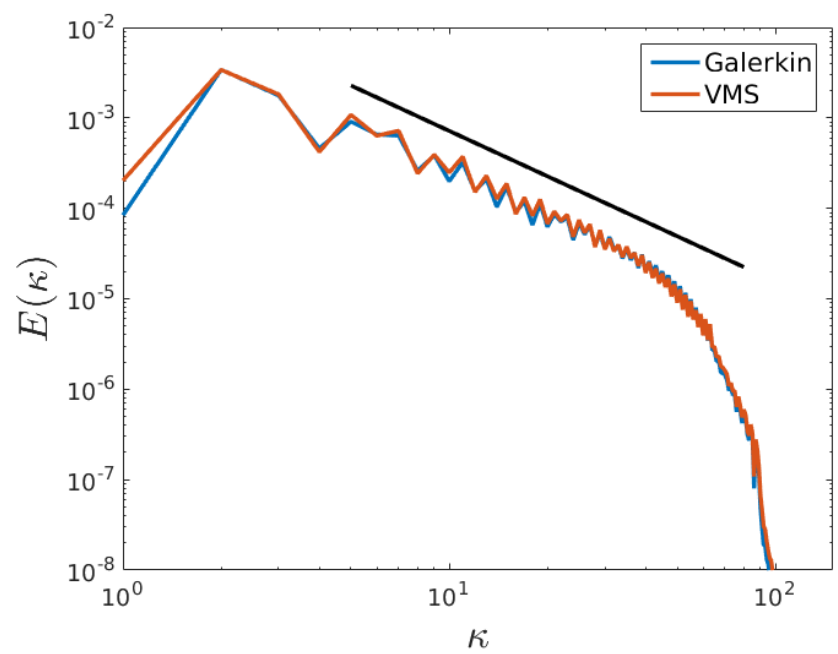

Figure 6: Taylor-Green vortex flow at $\mathrm{Re}=1600$. Energy spectra.

Remark As a semi-implicit method is used to treat nonlinear terms explicitly while evolving the system in time, a linear system governing a generalized Stokes problem must be solved at each time-step. Instead of addressing the full velocity-pressure system, we instead solve a reduced system involving only the divergence-free velocity field. This is made possible by relating the divergence- 


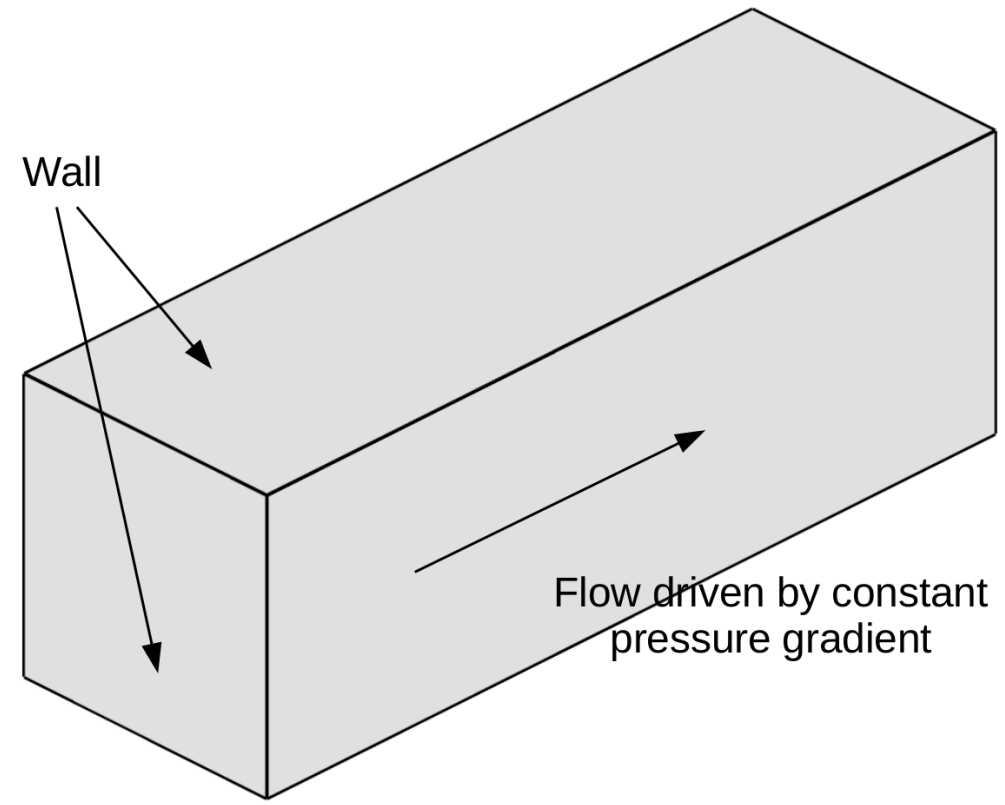

Figure 7: Turbulent channel flow at $\operatorname{Re}_{\tau}=395$. Problem setup.

free velocity field to a curl-conforming vector potential field. This yields a symmetric positive semi-definite system which may be efficiently solved using multigrid-preconditioned conjugate gradient methods. The discrete pressure field at a particular time instance may then be obtained by solving a discrete Laplace problem, though we note that the pressure field is not required for system solution as the velocity and pressure fields are completely decoupled in the Darcy-Dirichlet model.

\subsection{Turbulent channel flow at $\mathbf{R e}_{\tau}=395$}

We simulate turbulent channel flow at $\operatorname{Re}_{\tau}=395$. Here $\operatorname{Re}_{\tau}=u_{\tau} \delta / \nu$ at a friction-velocity-based Reynolds number, where $u_{\tau}=\sqrt{\tau_{w} / \rho}$ is the friction velocity, $\tau_{w}$ is the wall shear stress, and $\delta$ is the channel half width. The computational setup is shown in Figure 7, and the problem data is given in non-dimensional units. The problem domain is a rectangular box with dimensions $2 \pi \times 2 \times \frac{2}{3} \pi$ discretized using an unstretched mesh of $32^{3}$ elements. The flow is driven by a constant pressure gradient $f_{x}=1.472 \times 10^{-4}$ acting along the streamwise direction, prescribed as a known body force. This value of the pressure gradient results in the bulk streamwise velocity of unity. At the boundaries we employ: strongly-enforced no-penetration and weakly-enforced no-slip boundary conditions at the walls; periodic boundary conditions in the streamwise direction; and no-penetration boundary conditions in the spanwise direction. The flow equations are advanced 
in time using the generalized- $\alpha$ method $[19,38]$ with $\Delta t=0.025$. To achieve the desired $\operatorname{Re}_{\tau}$, the non-dimensional viscosity is set to $\nu=3.072 \times 10^{-5}$.

Two simulations of this flow are performed, using: 1 . The newly proposed divergence-conforming VMS formulation with the fine scales approximated by the element-level discrete Darcy-Dirichlet problem given by Eq. (39); 2. The original RBVMS formulation given by Eq. (45). In conjunction with the divergence-conforming VMS formulation, divergence-conforming B-splines with polynomial order $k=1$ are used. The RBVMS formulation makes use of the $C^{1}$-continuous quadratic B-spline discretization for all velocity and pressure unknowns. Note that the formal order of accuracy of the divergence-conforming B-spline discretization one order lower than RBVMS in this case.

Remark The Galerkin formulation with divergence-conforming B-splines failed to converge for this problem. This is likely caused by the well-known instability of the Galerkin technique in the regime of local convection dominance [15], which is not addressed by the divergence-conforming B-spline discretization alone. Galerkin may be rendered stable if the mesh is sufficiently refined, however, this direction was not pursued here.

Remark In the case of divergence-conforming VMS, the linear equation systems arising in the fine-scale problem on each element are solved directly, while the recently-proposed bi-partitioned iterative solution strategy [26] is used solve the linear equation systems arising in the global problem. The bi-partitioned approach is able to robustly handle the challenges associated with the lack of the "pressure-pressure block" in the linear-equation system arising in the divergence-conforming VMS discretization. The present simulations, as well as those reported in [26], illustrate the superior performance of the bi-partitioned linear-equation solver for incompressible-flow problems.

Flow statistics for both simulations are shown in Figure 8, where the data from the Direct Numerical Simulation (DNS) of this flow [41] is plotted for comparison. In the plots, the standard + units are employed, where $U^{+}=u / u_{\tau}$ and $y^{+}=y u_{\tau} / \nu$.

The streamwise turbulent fluctuations are overpredicted in the divergence-conforming VMS simulation relative to the equal-order RBVMS and DNS. This is not a surprising result, since discretizations of lower order and continuity tend to overpredict streamwise-velocity fluctuations relative to their higher-order and higher-continuity counterparts in under-resolved wall-bounded turbulent flows (see, e.g., [5, 2]). In fact, this is a common phenomenon in wall-bounded LES, and stems from the fact that LES cannot resolve the most energetic scales of the turbulence near the wall. The rms of real turbulent velocity field projected onto the LES space would be lower than that in the DNS, so the energy in the turbulence structures appears at the wrong scales in the LES. This issue tends to be more pronounced for lower-order discretizations, which we are also seeing in the present results.

On the other hand, the mean velocity in the divergence-conforming VMS is slightly more accurate near the channel center than in the RBVMS. Essentially, this indicates that for the same applied pressure gradient the flow rate in the RBVMS case is slightly lower than it should be, and 

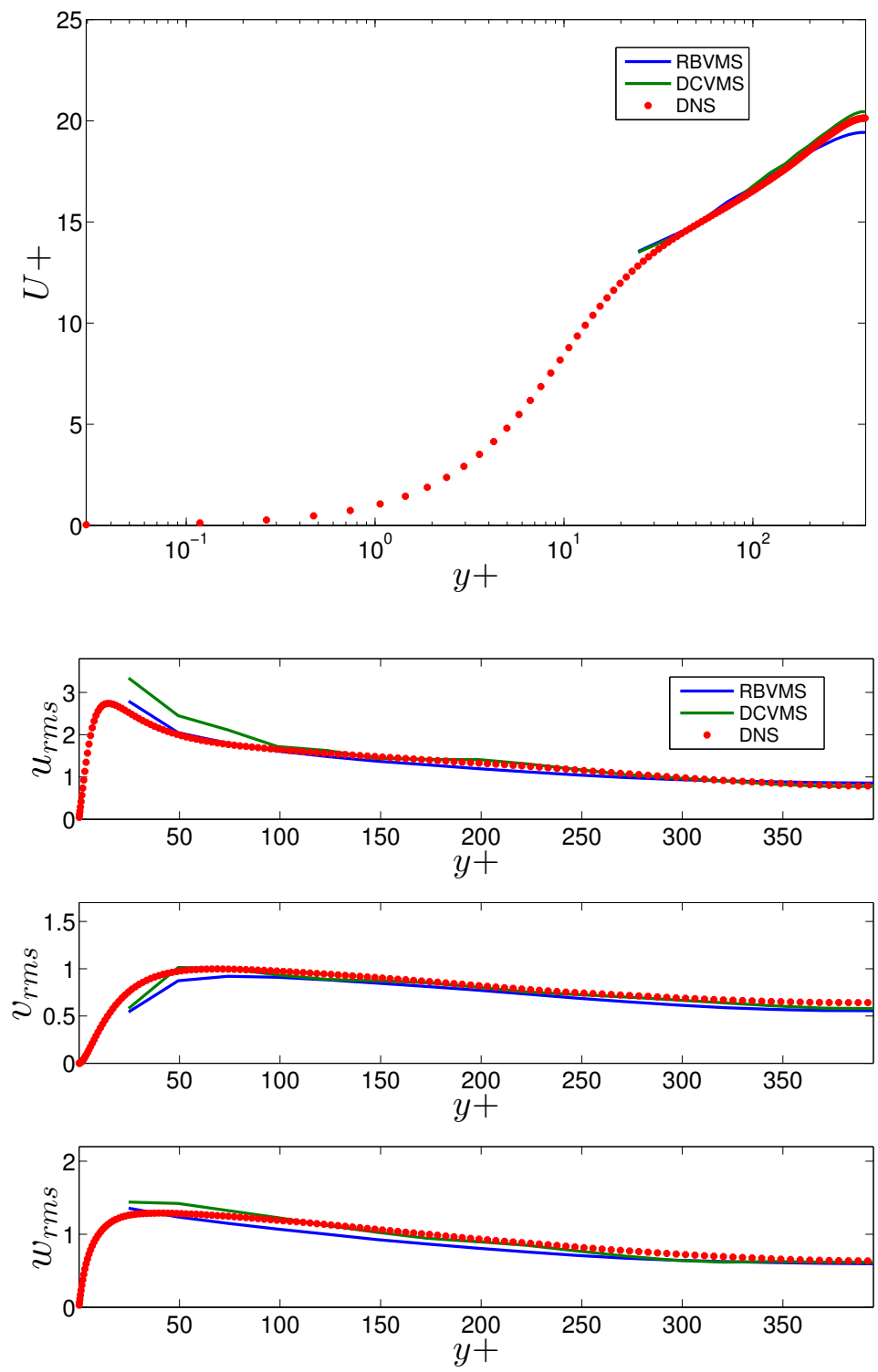

Figure 8: Statistics for $R e_{\tau}=395$ channel flow. Top: mean streamwise velocity. Bottom: root mean square of the velocity fluctuations.

suggests that in the RBVMS case the wall shear stress for a given flow rate is slightly overpredicted. The reader nevertheless should keep in mind that the results for both divergence-conforming VMS 


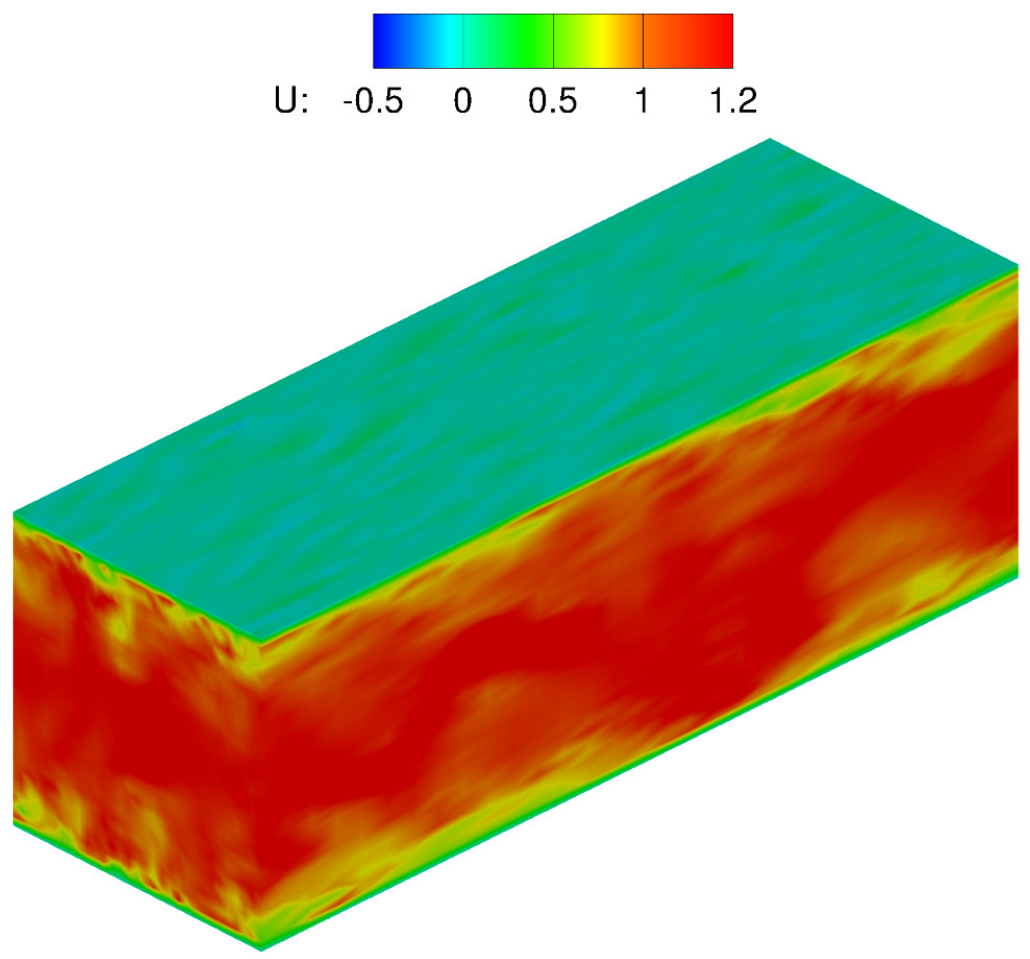

Figure 9: Turbulent channel flow at $R e_{\tau}=395$. Streamwise velocity contours.

and RBVMS are very accurate for the level of resolution employed in this problem.

Perhaps the most striking result of this study is the high accuracy levels the divergenceconforming VMS formulation is able to achieve despite the fact that the formal order of accuracy employed in the present computation is one less than in the RBVMS simulation.

Figure 9 shows instantaneous streamwise-velocity contours from the divergence-conforming VMS simulation. Weak enforcement of the no-slip conditions at the walls and a relatively coarse boundary-layer mesh give rise to non-negligible velocity fluctuations at no-slip walls (see top surface of the domain). However, this does not compromise the overall accuracy of the simulations.

\section{Conclusions}

In this paper we propose a new VMS formulation for incompressible turbulent flows, which employs the divergence-conforming B-spline discretization, and which, while $H^{1}$-compatible, also delivers a pointwise divergence-free discrete velocity solution. To ensure consistency of the velocity fine scales with the zero-divergence constraint, the discrete fine-scale problem is cast as an element- 
level Darcy problem posed over a restriction of the divergence-conforming B-spline basis to each individual element. The fine-scale Darcy problem is driven by the residual of the coarse scales, which retains consistency of the original RBVMS technique. Furthermore, the inverse of the stabilization parameter $\tau$ is interpreted as the diffusivity parameter of the aforementioned Darcy problem. In this sense, the stabilization parameter $\tau$ may be interpreted as an intrinsic time-scale associated with motion of subgrid vortices. This physical interpretation provides a connection between the mathematical framework of VMS modeling and standard physics-based LES approaches which we anticipate may be exploited to develop physics-based VMS models in the future. Strong enforcement of the normal-velocity component and weak enforcement of the tangential-velocity components are employed as part of the proposed formulation. Weak enforcement of tangential boundary and interface conditions presents a natural framework for divergence-conforming B-spline formulations, with the added benefit of improved solution accuracy in the cases of unresolved or marginally-resolved turbulent boundary layers. The numerical results indicate optimal convergence of the proposed formulation. Furthermore, lowest-order divergence-conforming discretizations yield correct spectral behavior in numerical simulation of decaying homogeneous isotropic turbulence, and they also lead to excellent accuracy of the turbulent flow statistics for turbulent channel flow, both for mean velocity as well as for velocity fluctuations.

The implementation of the divergence-conforming VMS formulation is obviously more involved than that of RBVMS using equal-order velocity-pressure discretization. This is largely due to the fact that a different approximation space needs to be programmed for each velocity-field component as well as for the pressure. Because in 3D four different basis-function sets needs to be evaluated, the time involved in the computation of element-level matrices and vectors is increased. In addition, because no pressure-pressure coupling in the left-hand-side matrix is present as in the case of PSPG or equal-order RBVMS formulations, a more sophisticated preconditioning technique is needed to solve the resulting linear-equation systems (like the recently-proposed bi-partitioned iterative solution strategy [26]). This results in additional implementation effort and possible increase in the computational cost compared to the equal-order case. Nevertheless, we feel that the accuracy benefits brought about by the divergence-conforming VMS formulation outweigh the above implementation challenges and increased computational cost, especially as the divergenceconforming technology matures going forward.

\section{Acknowledgments}

TO and TK acknowledge the financial support from the Norwegian Research Council and the industrial partners of the FSI-WT (216465/E20, http://www .fsi-wt.no) and NOWITECH: Norwegian Research Centre for Offshore Wind Technology (193823/S60, http://www.nowitech.no) projects. YB was partially supported by ARO Award W911NF-14-1-0296, while JY was partially supported by NSF CAREER Award. JE and CC were partially supported by the Air Force Office of Scientific Research under Grant No. FA9550-14-1-0113. The 2D numerical examples were computing using 
the nutils.org software framework.

\section{References}

[1] I. Akkerman, Y. Bazilevs, D. J. Benson, M. W. Farthing, and C. E. Kees. Free-surface flow and fluid-object interaction modeling with emphasis on ship hydrodynamics. Journal of Applied Mechanics, 79:010905, 2012.

[2] I. Akkerman, Y. Bazilevs, V. M. Calo, T. J. R. Hughes, and S. Hulshoff. The role of continuity in residual-based variational multiscale modeling of turbulence. Computational Mechanics, 41:371-378, 2008.

[3] I. Babuška. The finite element method with Lagrange multipliers. Numer. Math., 20(179-192), 1973.

[4] Y. Bazilevs and I. Akkerman. Large eddy simulation of turbulent Taylor-Couette flow using isogeometric analysis and the residual-based variational multiscale method. Journal of Computational Physics, 229:3402-3414, 2010.

[5] Y. Bazilevs, V. M. Calo, J. A. Cottrell, T. J. R. Hughes, A. Reali, and G. Scovazzi. Variational multiscale residual-based turbulence modeling for large eddy simulation of incompressible flows. Computer Methods in Applied Mechanics and Engineering, 197:173-201, 2007.

[6] Y. Bazilevs and T. J. R. Hughes. Weak imposition of Dirichlet boundary conditions in fluid mechanics. Computers and Fluids, 36:12-26, 2007.

[7] Y. Bazilevs and T. J. R. Hughes. NURBS-based isogeometric analysis for the computation of flows about rotating components. Computational Mechanics, 43:143-150, 2008.

[8] Y. Bazilevs, C. Michler, V. M. Calo, and T. J. R. Hughes. Weak Dirichlet boundary conditions for wall-bounded turbulent flows. Computer Methods in Applied Mechanics and Engineering, 196:4853-4862, 2007.

[9] Y. Bazilevs, C. Michler, V. M. Calo, and T. J. R. Hughes. Isogeometric variational multiscale modeling of wall-bounded turbulent flows with weakly enforced boundary conditions on unstretched meshes. Computer Methods in Applied Mechanics and Engineering, 199:780-790, 2010 .

[10] Y Bazilevs, J Yan, M de Stadler, and S Sarkar. Computation of the flow over a sphere at $\mathrm{Re}=3,700$ : A comparison of uniform and turbulent inflow conditions. Journal of Applied Mechanics, 81(12):121003, 2014. 
[11] M Brachet, D Meiron, B Nickel, R Morf, and U Frisch. Small-scale structure of the taylor-green vortex. Journal of Fluid Mechanics, 130:411-452, 1983.

[12] F. Brezzi and M. Fortin. Mixed and Hybrid Finite Element Methods. Springer-Verlag, Berlin, 1991.

[13] F. Brezzi, L. P. Franca, T. J. R. Hughes, and A. Russo. $b=\int$ g. Computer Methods in Applied Mechanics and Engineering, 145:329-339, 1997.

[14] F. Brezzi and A. Russo. Choosing bubbles for advection-diffusion problems. Mathematical Models and Methods in Applied Science, 4:571-587, 1994.

[15] A. N. Brooks and T. J. R. Hughes. Streamline upwind/Petrov-Galerkin formulations for convection dominated flows with particular emphasis on the incompressible Navier-Stokes equations. Computer Methods in Applied Mechanics and Engineering, 32:199-259, 1982.

[16] A. Buffa, C. de Falco, and G. Sangalli. Isogeometric analysis: Stable elements for the 2D Stokes equation. International Journal for Numerical Methods in Fluids, 65:1407-1422, 2011.

[17] A. Buffa, J. Rivas, G. Sangalli, and R. Vazquez. Isogeometric discrete differential forms in three dimensions. SIAM Journal on Numerical Analysis, 49:818-844, 2011.

[18] V.M. Calo. Residual-based multiscale turbulence modeling: finite volume simulations of bypass transition. PhD thesis, Stanford University, 2004.

[19] J. Chung and G. M. Hulbert. A time integration algorithm for structural dynamics withimproved numerical dissipation: The generalized- $\alpha$ method. Journal of Applied Mechanics, 60:371-75, 1993.

[20] J. A. Cottrell, T. J. R. Hughes, and Y. Bazilevs. Isogeometric Analysis. Toward Integration of $C A D$ and FEA. Wiley, 2009.

[21] M.G. Cox. The numerical evaluation of B-splines. Technical report, National Physics Laboratory DNAC 4, 1971.

[22] M.A. Cruchaga. A study of the backward-facing step problem using a generalized streamline formulation. Communications in Numerical Methods in Engineering, 14:697-708, 1998.

[23] C. de Boor. On calculation with B-splines. Journal of Approximation Theory, 6:50-62, 1972.

[24] C. de Boor, T. Lyche, and L.L. Schumaker. On calculating with B-splines II. integration. Numerische Methoden der Approximationstheorie, 30:123-146, 1976.

[25] E. Erturk. Numerical solutions of 2-D steady incompressible flow over a backward-facing step, part I: high Reynolds number solutions. Computers and Fluids, pages 633-655, 2008. 
[26] Mahdi Esmaily-Moghadam, Yuri Bazilevs, and Alison L Marsden. A bi-partitioned iterative algorithm for solving linear systems arising from incompressible flow problems. Computer Methods in Applied Mechanics and Engineering, 286:40-62, 2015.

[27] J.A. Evans and T.J.R. Hughes. Isogeometric divergence-conforming B-splines for the Darcy-Stokes-Brinkman equations. Mathematical Models and Methods in Applied Sciences, 23(4):671-741, 2013.

[28] J.A. Evans and T.J.R. Hughes. Isogeometric divergence-conforming B-splines for the steady Navier-Stokes equations. Mathematical Models and Methods in Applied Sciences, 23(8):14211478 1421-1478, 2013.

[29] J.A. Evans and T.J.R. Hughes. Isogeometric divergence-conforming B-splines for the unsteady Navier-Stokes equations. Journal of Computational Physics, 241:141-167, 2013.

[30] Roozbeh Golshan, Andrés E Tejada-Martínez, Mario Juha, and Yuri Bazilevs. Large-eddy simulation with near-wall modeling using weakly enforced no-slip boundary conditions. Computers and Fluids, 118:172-181, 2015.

[31] Ming-Chen Hsu, I. Akkerman, and Y. Bazilevs. Finite element simulation of wind turbine aerodynamics: Validation study using NREL Phase VI experiment. Wind Energy, 17:461-481, 2014 .

[32] Ming-Chen Hsu and Y. Bazilevs. Fluid-structure interaction modeling of wind turbines: simulating the full machine. Computational Mechanics, 50:821-833, 2012.

[33] Ming-Chen Hsu, Y. Bazilevs, V. M. Calo, T. E. Tezduyar, and T. J. R. Hughes. Improving stability of stabilized and multiscale formulations in flow simulations at small time steps. Computer Methods in Applied Mechanics and Engineering, 199:828-840, 2010.

[34] T. J. R. Hughes. Multiscale phenomena: Green's functions, the Dirichlet-to-Neumann formulation, subgrid scale models, bubbles, and the origins of stabilized methods. Computer Methods in Applied Mechanics and Engineering, 127:387-401, 1995.

[35] T. J. R. Hughes, J. A. Cottrell, and Y. Bazilevs. Isogeometric analysis: CAD, finite elements, NURBS, exact geometry, and mesh refinement. Computer Methods in Applied Mechanics and Engineering, 194:4135-4195, 2005.

[36] T.J.R. Hughes, L. Mazzei, and K.E. Jansen. Large eddy simulation and the variational multiscale method. Computing and Visualization Science, 3:47-59, 2000.

[37] T.J.R. Hughes and G. Sangalli. Variational multiscale analysis: the fine-scale Green's function, projection, optimization, localization and stabilized methods. SIAM Journal on Numerical Analysis, 45(2):539-557, 2007. 
[38] K. E. Jansen, C. H. Whiting, and G. M. Hulbert. A generalized- $\alpha$ method for integrating the filtered Navier-Stokes equations with a stabilized finite element method. Computer Methods in Applied Mechanics and Engineering, 190:305-319, 2000.

[39] K.A. Johannessen, M. Kumar, and T. Kvamsdal. Divergence-conforming discretization for Stokes problem on locally refined meshes using LR B-splines. Computer Methods in Applied Mechanics and Engineering, 293:38-70, 2015.

[40] J.M. Leone Jr. Open boundary condition symposium benchmark solution: stratified flow over a backward-facing step. International Journal for Numerical Methods in Fluids, 11:969-984, 1990 .

[41] Robert D Moser, John Kim, and Nagi N Mansour. Direct numerical simulation of turbulent channel flow up to Re=590. Phys. Fluids, 11(4):943-945, 1999.

[42] J. Nitsche. Über ein variationsprinzip zur lösung von Dirichlet-Problemen bei verwendung von teilräumen, die keinen randbedingungen unterworfen sind. Abhandlungen aus dem mathematischen Seminar der Universität Hamburg, 36(1):9-15, 1971.

[43] S. B. Pope. Turbulent Flows. Cambridge University Press, Cambridge, 2000.

[44] T.M. Shih, C.H. Tan, and B.C. Hwang. Effects of grid staggering on numerical schemes. International Journal for Numerical Methods in Fluids, 9:193-212, 1989.

[45] T. Tezduyar and S. Sathe. Stabilization parameters in SUPG and PSPG formulations. Journal of Computational and Applied Mechanics, 4:71-88, 2003.

[46] T. E. Tezduyar. Computation of moving boundaries and interfaces and stabilization parameters. International Journal for Numerical Methods in Fluids, 43:555-575, 2003.

[47] T. E. Tezduyar. Finite element methods for fluid dynamics with moving boundaries and interfaces. In E. Stein, R. De Borst, and T. J. R. Hughes, editors, Encyclopedia of Computational Mechanics, Volume 3: Fluids, chapter 17. John Wiley \& Sons, 2004.

[48] T. E. Tezduyar, S. Mittal, S. E. Ray, and R. Shih. Incompressible flow computations with stabilized bilinear and linear equal-order-interpolation velocity-pressure elements. Computer Methods in Applied Mechanics and Engineering, 95:221-242, 1992.

[49] D. C. Wilcox. Turbulence Modeling for CFD. DCW Industries, La Canada, CA, 1998. 


\section{A Appendix: Exact representation of the discrete stream function}

In this appendix we exactly reconstruct the stream function corresponding to our discrete solution, exploiting the facts that 1) our approximation is pointwise divergence free; and 2) derivatives of B-splines are again splines. This construction works for affine transformations from parameter space and is performed patchwise. For simplicity, we consider the $2 \mathrm{D}$ case and denote the velocity approximation by $\left\{u^{h}, v^{h}\right\}$ and the gradient by $\{\partial / \partial x, \partial / \partial y\}$. The fact that the approximation is pointwise div-conforming implies that there exists a stream function $\psi^{h}$ (unique up to a constant) which exactly describes it:

$$
u^{h}=\frac{\partial \psi^{h}}{\partial y}, \quad v^{h}=-\frac{\partial \psi^{h}}{\partial x} .
$$

To find $\psi^{h}$, we find the lower left corner of the patch, $(X, Y)$, and integrate the first of (55) w.r.t. the second argument

$$
\psi^{h}(x, y)=\psi^{h}(x, Y)+\int_{Y}^{y} u^{h}(x, \tau) d \tau
$$

and take the derivative w.r.t. the first argument (noting that, by definition, $\partial u^{h} / \partial x=-\partial v^{h} / \partial y$ )

$$
\begin{aligned}
\frac{\partial \psi^{h}}{\partial x}(x, y) & =\frac{\partial \psi^{h}}{\partial x}(x, Y)+\int_{Y}^{y}-\frac{\partial v^{h}}{\partial y}(x, \tau) d \tau \\
& =\frac{\partial \psi^{h}}{\partial x}(x, Y)+v^{h}(x, Y)-v^{h}(x, y) \\
& =-v^{h}(x, y),
\end{aligned}
$$

where the last equality follows from (55). Thus,

$$
\frac{\partial \psi^{h}}{\partial x}(x, Y)=-v^{h}(x, Y)
$$

which integrates to

$$
\psi^{h}(x, Y)=\psi^{h}(X, Y)-\int_{X}^{x} v^{h}(\tau, Y) d \tau
$$

This can be substituted into (56) to find

$$
\psi^{h}(x, y)=\psi^{h}(X, Y)+\int_{Y}^{y} u^{h}(x, \tau) d \tau-\int_{X}^{x} v^{h}(\tau, Y) d \tau,
$$

where the constant $\psi^{h}(X, Y)$ is arbitrary and can be used for inter-patch continuity. In the sequel, it is set to zero.

The second step is to express the integrals in (57) in terms of splines for exact evaluation at arbitray points $(x, y)$ inside the patch. Because the map from parameter space is affine, we can for 
simplicity assert that the map from parameter space to $(x, y)$ is identity. We adopt the notation from $\S 3$ except that it is more convenient to work exclusively with knot vectors $\Xi+\in \mathbb{R}^{m+2 p+3}$ and $H^{+} \in \mathbb{R}^{n+2 p+3}$, so we omit the superscript. Note that these knot vectors are open w.r.t. the order $p+1$ splines which compose $\psi^{h}$. We have

$$
\begin{aligned}
& u^{h}(x, y)=s_{i, p+1}^{\Xi}(x) s_{j, p}^{H}(y) \hat{u}_{i j}^{h}, \quad i<m+p+1, \quad j<n+p+2, \\
& v^{h}(x, y)=s_{i, p}^{\Xi}(x) s_{j, p+1}^{H}(y) \hat{v}_{i j}^{h}, \quad i<m+p+2, \quad j<n+p+1 .
\end{aligned}
$$

We have in this case

$$
s_{0, p}^{\Xi}=0=s_{m+p-1, p}^{\Xi} \quad \text { and } \quad s_{0, p}^{H}=0=s_{n+p-1, p}^{H}
$$

because of $(p+2)$-fold repeated first and last knots. We can find an explicit expression for $\psi^{h}$ due to the following lemma.

Lemma 1. The integrals of (57) can be written as

$$
\begin{aligned}
& \int_{Y}^{y} u^{h}(x, \tau) d \tau=s_{i, p+1}^{\Xi}(x) \underbrace{\int_{Y}^{y} s_{j, p}^{H}(\tau) d \tau}_{=: A_{j k}^{H} s_{k, p+1}^{H}(y)} \hat{u}_{i j}^{h} \\
& \int_{X}^{x} v^{h}(\tau, Y) d \tau=\underbrace{\int_{X}^{x} s_{j, p}^{\Xi}(\tau) d \tau}_{=: A_{j k}^{\Xi} s_{k, p+1}^{\Xi}(x)} s_{i, p+1}^{H}(Y) \hat{v}_{i j}^{h}
\end{aligned}
$$

where, for $(Z, n, p) \in\left\{\left(\Xi, n_{\Xi}, p\right),\left(H, n_{H}, p\right)\right\}, A^{Z} \in \mathbb{R}^{(n+p+2) \times(n+p+1)}$ is given by

$$
A^{Z}:=\frac{1}{p+1}\left[\begin{array}{ccccc}
0 & 0 & 0 & \cdots & 0 \\
& d_{1} & d_{1} & \cdots & d_{1} \\
& & d_{2} & \cdots & d_{2} \\
& & & \ddots & \vdots \\
& & & & d_{n+p} \\
0 & 0 & 0 & \cdots & 0
\end{array}\right]
$$

with $d_{j}=Z_{j+p+1}-Z_{j}$.

Proof. The differentiation formula for spline functions is

$$
\frac{d}{d \xi} s_{j, p+1}^{Z}(\xi)=\frac{(p+1) s_{j, p}^{Z}(\xi)}{Z_{j+p+1}-Z_{j}}-\frac{(p+1) s_{j+1, p}^{Z}(\xi)}{Z_{j+p+2}-Z_{j+1}}, \quad j<n+p+1,
$$

which; with the notation $S_{p}^{Z}:=\left\{s_{j, p}^{Z}\right\}_{j<n+p+2}$ (the first and last entries of which vanish acc. to eq. 58) and $S_{p+1}^{Z}:=\left\{s_{j, p+1}^{Z}\right\}_{j<n+p+1}$; can be written as

$$
\frac{d}{d \xi} S_{p+1}^{Z}(\xi)=M^{Z} S_{p}^{Z}(\xi)
$$


with $M^{Z} \in \mathbb{R}^{(n+p+1) \times(n+p+2)}$ given by

$$
M^{Z}:=(p+1)\left[\begin{array}{ccccc}
d_{0}^{-1} & -d_{1}^{-1} & & & \\
& d_{1}^{-1} & -d_{2}^{-1} & & \\
& & \ddots & \ddots & \\
& & & d_{n+p}^{-1} & -d_{n+p+1}^{-1}
\end{array}\right]
$$

It is easily verified that

$$
A_{i k}^{Z} M_{k j}^{Z}= \begin{cases}0, & j \in\{0, n+p+1\}, \\ \delta_{i j}, & 0<j<n+p+1 ;\end{cases}
$$

where the first case corresponds to the zero functions of (58). Therefore, by integrating (60),

$$
\int_{Z_{0}}^{\xi} S_{p}^{Z}(\tau) d \tau=A^{Z} S_{p+1}^{Z}(\xi)
$$

(cf. [24]) which can be used to eliminate the integrals as asserted by the lemma.

Using lemma 1, the stream function can be written as

$$
\psi^{h}(x, y)=s_{i, p+1}^{\Xi}(x)\left(A_{k j}^{H} s_{j, p+1}^{H}(y) \hat{u}_{i k}^{h}-A_{k i}^{\Xi} s_{j, p+1}^{H}(Y) \hat{v}_{k j}^{h}\right) .
$$

Because the basis $S_{p+1}^{N}$ possesses the partition-of-unity property and is interpolatory at $y=Y$, the second term can be simplified using $s_{j, p+1}^{H}(Y) \hat{v}_{k j}^{h}=s_{j, p+1}^{H}(y) \hat{v}_{k 0}^{h} 1_{j}$, where the factor $1_{j}=1$ simply serves to preserve summation over $j$, such that

$$
\psi^{h}(x, y)=s_{i, p+1}^{\Xi}(x) s_{j, p+1}^{H}(y)\left(A_{k j}^{H} \hat{u}_{i k}^{h}-A_{k i}^{\Xi} \hat{v}_{k 0}^{h} 1_{j}\right) .
$$

It is interesting to see that the number of degrees of freedom determining $\psi^{h}$, namely $(m+p+$ $1) \times(n+p)$ from $\hat{u}$ and $m+p$ from $\hat{v}$, is precisely one less than the dimension of the space $\mathbb{S}^{p+1, p+1}(\Xi, H) \ni \psi^{h}$. This "missing" degree of freedom of course coincides with the arbitrary constant. Of course, one could interchange the roles of $u^{h}$ and $v^{h}$ in the above derivation and arrive at the equivalent representation

$$
\psi^{h}(x, y)=s_{i, p+1}^{\Xi}(x) s_{j, p+1}^{H}(y)\left(A_{k j}^{H} \hat{u}_{0 k}^{h} 1_{i}-A_{k i}^{\Xi} \hat{v}_{k j}^{h}\right) .
$$

